Pacific Journal of Mathematics

HOMOLOGY OF COVERINGS 


\title{
HOMOLOGY OF COVERINGS
}

\author{
JOHN HEMPEL
}

\begin{abstract}
This paper deals with an analysis of the first homology of a finite sheeted covering space of a complex and gives applications to some questions about 3-manifolds. Section 2 considers the relation between the property that a 3-manifold be virtually Haken and the, seemingly stronger, property that some finite sheeted cover has positive first betti number. Section 3 gives a procedure for computing the homology of a finite cover in terms of a presentation of the fundamental group of the base, and its action on the fiber and includes generalizations of the Fox-Goeritz theorem for cyclic covers to arbitrary abelian covers and to dihedral covers. Section 4 applies these theorems to 3-manifolds which have various types of symmetry and include some conditions which guarantee finite covers with positive first betti number. The paper concludes with a section of examples.
\end{abstract}

1. Introduction. The homology groups of the various covering spaces of a space $M$ are of interest for a variety of reasons. For one thing, when restricted to some "characteristic" collection of coverings (the cyclic coverings of a knot space, for example) they provide invariants of the base which are much more tractable than its homotopy groups and, in general, much richer than its homology groups.

There is another point of particular interest to the study of 3-dimensional manifolds which we proceed to describe.

Following $[\mathbf{J}]$ we use the term Haken manifold to mean a compact, orientable, irreducible 3-manifold which is sufficiently large in the sense that it contains a 2-sided incompressible surface. The study of 3-manifolds splits nicely into the cases of finite fundamental groups and infinite fundamental groups. The vast majority of what is known in the second case - determination by homotopy type, existence of geometric structures, the homeomorphism and classification problems, etc. - is established only for Haken manifolds as can be discovered in the recent works of Jaco-Shalen [JS], Johannson $\left[\mathbf{J}_{\mathbf{0}}\right]$, Thurston [T], and the nice summary by Waldhausen $\left[\mathbf{W}_{3}\right]$.

The examples of compact, orientable, irreducible 3-manifolds with infinite fundamental groups which are not Haken manifolds $\left[\mathbf{W}_{\mathbf{1}}\right],[\mathbf{C J R}]$, $[\mathbf{F H}],[\mathbf{H T}]$ seem to have the property that some finite sheeted cover is a Haken manifold. We will say that such a manifold is virtually Haken. This is consistent with the terminology from group theory which says that a 
group is virtually of some class $\mathcal{C}$ (e.g. solvable) of groups if it has a subgroup of finite index in the class $\mathcal{C}$. The term almost sufficiently large has also been used.

The following question has been raised by Thurston, Waldhausen and others.

1.1 Question. If $M$ is a compact, orientable irreducible 3-manifold with $\pi_{1}(M)$ infinite, is $M$ virtually Haken?

Since any compact 3-manifold $M$, with $\beta_{1}(M)=\operatorname{rank} H_{1}(M ; \mathbf{Z})>0$, contains a 2-sided incompressible surface (cf. $\left[\mathbf{H}_{1}\right]$, Lemma 6.6), we ask

1.2 Question. If $M$ is a compact, orientable, irreducible 3-manifold with $\pi_{1}(M)$ infinite, is $\pi_{1}(M)$ virtually representable onto $\mathbf{Z}$ ?

The condition means that there is a finite cover $\tilde{M} \rightarrow M$ with $\beta_{1}(\tilde{M})$ $>0$, and clearly an affirmative answer to 1.2 implies an affirmative answer to 1.1. At first glance 1.2 may appear too strong, since there are Haken manifolds with $\beta_{1}(M)=0$, and which, therefore, only contain separating incompressible surfaces. However, such separating surfaces often are covered, in some finite sheeted cover of $M$, by nonseparating surfaces. Numerous examples of this are provided by 2.5 , as well as by the Seifert fibered spaces $\left[\mathbf{W}_{\mathbf{1}}\right]$, which all have finite covers by circle bundles over a surface. It is natural to ask if this is always the case:

1.3 Question. If $M$ is a Haken manifold, is $\pi_{1}(M)$ virtually representable onto $\mathbf{Z}$ ?

In $\$ 2$ we investigate this question further - paying particular attention to the case of manifolds which are the union (along their incompressible boundaries) of two knot spaces. The complement, in $S^{3}$, of an open regular neighborhood of a knot is called a knot space.

We show that the answer to 1.3 is always yes for the union of two torus knot spaces (Corollary 2.5) and discuss means for resolving this question for other knots.

In $\S 3$ we consider the problem of computing $H_{1}(\tilde{M})$ for an arbitrary finite sheeted cover $p: \tilde{M} \rightarrow M$ of a finite CW complex from a presentation of $\pi_{1}(M)$. There is a combinatorial procedure for doing this introduced in $\left[\mathbf{F}_{3}\right]$ which we present in Theorem 3.1. Beginning with a presentation of $\pi_{1}(M)$ with, say, $m$ generators and applied to a $q$-sheeted covering, one is led to an $m q \times m q$ matrix of integers which presents $H_{1}(\tilde{M}) \oplus \mathbf{Z}^{q-1}$. 
Unfortunately even in cases of moderate complexity this becomes too large to be of much value and the procedure appears to have been largely neglected in general. However, in the case of finite cyclic covers (i.e., regular with cyclic covering group) there are elegant tricks for simplifying the situation, and much has been written on the subject, particularly as it applies to the cyclic covers of knots and links as in the Goeritz-Fox theorem $[\mathbf{G}],\left[\mathbf{F}_{3}\right]$ with improvements by Gordon $\left[\mathbf{G}_{\mathbf{0}}\right]$, Hosokawa-Kinoshita $[\mathrm{HK}]$, and others.

Put in the proper setting, these results generalize to any finite, abelian (regular) cover $\tilde{M}$, of any $\mathrm{CW}$ complex $M$, as we present in Theorem 3.5. The $m q \times m q$ matrix of integers can be replaced by $q m \times m$ matrices with entries in the extension of $\mathbf{Z}$ by the $q$ th roots of unity which, modulo $q$-torsion, also presents $H_{1}(M)$. This gives a computational simplification and has theoretical interest as well, since the Galois group of the extension permutes these matrices. Thus, for example, $\beta_{1}(\tilde{M}) \equiv \beta_{1}(\hat{M}) \bmod 2$ (Corollary 3.6) where $\hat{M} \rightarrow M$ corresponds to the elements of order two in $\operatorname{Aut}(M \rightarrow M)(\hat{M}=M$ if $q$ is odd $)$.

These techniques extend, somewhat, to other situations. In 3.7 we give a version which applies to an analysis of irregular dihedral covers of any complex, and in 3.8 we apply this to the 3-fold irregular branched covers of $S^{3}$ branched over a knot. Recall [Hi], [M] that every closed oriented 3-manifold can be so expressed.

In $§ 4$ we consider closed 3-manifolds which have a Heegaard splitting whose "sides" can be interchanged by an involution $\tau$ of the manifold and which we call ( \pm )-symmetric according as $\operatorname{deg} \tau= \pm 1$. These occur commonly as double branched or unbranched covers of other 3-manifolds perhaps with "conical singularities" as described further in this section. The fundamental group of such a 3-manifold has a presentation with a strong form of symmetry (Theorem 4.5). Applying the results of $\S 3$ to this presentation yields additional information about the homology of the finite coverings of such manifolds. The symmetry reflects itself in the abelian coverings of these manifolds as explained in Theorem 4.3. Regarding Question 1.3 we show that if $M$ has a (-)-symmetric splitting relative to an involution $\tau: M \rightarrow M$ and $H_{1}\left(M / \tau ; \mathbf{Z}_{2}\right) \neq 0$, then $\pi_{1}(M)$ is virtually representable to $\mathbf{Z}$ (Corollary 4.5 - see also Example 5.4).

In $\S 5$ we discuss a number of examples to illustrate the theorems.

In 5.2 we show that, for every $k$, the $3 k$-fold cyclic covers of $S^{3}$ branched over the figure eight knot has fundamental group virtually representable to $\mathbf{Z}$.

Examples 5.4 and 5.5 illustrate symmetric Heegaard splittings as well as the calculational techniques from $\S \S 3$ and 4. 
2. Coverings with positive first betti number. It is convenient to consider, for any space $X$, the class $\mathscr{F}(X)$ of normal subgroups of finite index in $\pi_{1}(X)$, and to note its functorality: For a map $f: X \rightarrow Y$ we have a function $\mathscr{F}(f): \mathscr{F}(Y) \rightarrow \mathscr{F}(X)$ given by $\mathscr{F}(f)(N)=f_{*}^{-1}(N)$.

The evidence which suggests Question 1.3 is that a separating incompressible surface in a 3-manifold $M$ is often covered, in some finite sheeted covering of $M$, by a nonseparating one. The conditions for this to occur are given by the following theorem.

2.1 TheOREM. Let the finite $C W$ complex $M$ be expressed as $M=M_{1}$ $\cup M_{2}$, where $M_{1}, M_{2}$, and $S=M_{1} \cap M_{2}$ are connected subcomplexes, and $S$ is bicollared in $M$. Then there is a finite sheeted covering $p: \tilde{M} \rightarrow M$ such that some component of $p^{-1}(S)$ is nonseparating in $\tilde{M}$ if and only if there are groups $N_{k} \in \mathscr{F}\left(M_{k}\right)(k=1,2)$ such that

(i) $\mathscr{F}\left(\eta_{1}\right)\left(N_{1}\right)=\mathscr{F}\left(\eta_{2}\right)\left(N_{2}\right), \eta_{k}: S \rightarrow M_{k}$ inclusion, and

(ii) $\eta_{k *}\left(\pi_{1}(S)\right) \cdot N_{k} \neq \pi_{1}\left(M_{k}\right)(k=1,2)$.

Proof. Suppose we are given $p: \tilde{M} \rightarrow M$ such that $p^{-1}(S)$ has a nonseparating component. We may assume $p$ is a regular cover; otherwise intersect the finitely many distinct conjugates of $p_{*} \pi_{1}(\tilde{M})$ and consider the corresponding regular cover.

Let $N_{k}=\mathscr{F}\left(\theta_{k}\right)\left(p_{*} \pi_{1}(\tilde{M})\right) \in \mathscr{F}\left(M_{k}\right)\left(\theta_{k}: M_{k} \rightarrow M\right.$ inclusion $)$.

Property (i) follows from functorality. For (ii) note that for any component $\tilde{M}_{k}$ of $p^{-1}\left(M_{k}\right), p_{*} \pi_{1}\left(\tilde{M}_{k}\right)=N_{k}$. Since some (hence, by regularity, every) component of $p^{-1}(S)$ is nonseparating, each component of $p^{-1}\left(M_{k}\right)$ must contain at least two components of $p^{-1}(S)$. However, the number of components of $p^{-1}(S)$ contained in a given component of $p^{-1}\left(M_{k}\right)$ is the index of $\eta_{k *} \pi_{1}(S) \cdot N_{k}$ in $\pi_{1}\left(M_{k}\right)$; so property (ii) follows.

Conversely, given the subgroups $N_{k}$, we form the corresponding finite sheeted regular covers $p_{k}: \tilde{M}_{k} \rightarrow M_{k}$. By (ii) $p_{k}^{-1}(S)$ has components $\tilde{S}_{k, 1}, \ldots, \tilde{S}_{k, n k}\left(n_{k}>1\right)$. By (i) $p_{1} \mid \tilde{S}_{1, i}$ and $p_{2} \mid \tilde{S}_{2, j}$ are equivalent coverings. We take $\left[n_{1}, n_{2}\right] / n_{1}$ copies of $\tilde{M}_{1},\left[n_{1}, n_{2}\right] / n_{2}$ copies of $\tilde{M}_{2}$ and identify, in some order, the copies of the $\tilde{S}_{1, i}$ with the copies of the $\tilde{S}_{2, j}$ by the equivalence mentioned above. The result will yield a cover $p: \tilde{M} \rightarrow M$, as desired. This cover will probably be irregular, but one may pass to a regular one, if desired, as in the first part.

We will say that $L \in \mathscr{F}(S)$ is proper modulo $\eta: S \rightarrow M$ if $L=\mathscr{F}(\eta)(N)$ for some $N \in \mathscr{F}(M)$ such that $N \cdot \eta_{*} \pi_{1}(S) \neq \pi_{1}(M)$ and, when $S \subset M$, we will denote by $\rho \mathscr{F}_{M}(S)$ the set of subgroups which are proper modulo 
the inclusion $S \hookrightarrow M$. Observe that properties (i) and (ii) of Theorem 2.1 can be abbreviated:

$$
\mathscr{P} \mathscr{F}_{M_{1}}(S) \cap \mathscr{P}_{\mathcal{F}_{M_{2}}}(S) \neq \varnothing
$$

Observe also that if $S$ is an incompressible torus in the boundary of a 3-manifold $M$, and if $N \in \mathcal{F}(M)$ is such that $\pi_{1}(M) / N$ is nonabelian, then $\eta_{*}^{-1}(N) \in \mathcal{P} \mathscr{F}_{M}(S)$.

In order to apply Theorem 2.1 to Problem 1.3 we are faced with

2.2 Problem. For $M$ a 3-manifold and $S$ an incompressible surface in $\partial M$, describe $\mathscr{P} \mathscr{F}_{M}(S)$.

We caution that this is a difficult problem. In case $M$ is a knot space and $S=\partial M$, a complete solution would presumably settle the question of whether the knot has property $P[\mathbf{S}]$ (or produce a 3-manifold $M$ for which $\pi_{1}(M)$ has no nontrivial finite quotients).

Nonetheless, the case in which $S$ is a torus is still the most tractable case of Problem 2.2 and we proceed to make some observations on it. For this we need a normal form for elements of $\mathscr{F}(S)$ which is easily given by

2.3 LEMMA. Let $\{\mu, \lambda\}$ be a free basis for a free abelian group $G$. Then a subgroup $H$ of finite index in $G$ is uniquely of the form $H=\operatorname{gp}\left\{\mu^{a}, \mu^{b} \lambda^{c}\right\}$ where $a, c \geq 1,0 \leq b<a$. Moreover, $H$ is fully invariant in $G$ if and only if $a=c$ and $b=0$.

Proof. $H=\operatorname{gp}\left\{\mu^{a}, \mu^{b} \lambda^{c}\right\}$, where $a$ is the index of $H \cap \operatorname{gp}\{\mu\}$ in $\operatorname{gp}\{\mu\}$, $c$ is the index of $H$ in $H \cdot \operatorname{gp}\{\mu\}$ and $b$ is the least nonnegative integer such that $\mu^{b} \lambda^{c} \in H$.

Since $\operatorname{gp}\left\{\mu^{a}, \lambda^{a}\right\}=H^{a}$, it is fully invariant, and a calculation shows that every fully invariant subgroup of finite index must have this form.

2.4 LEMMA. Let $M$ be $a(p, q)$ torus knot space and $a>2$ an integer relatively prime to both $p$ and $q$. Then $\mathcal{P}_{\mathcal{F}}(\partial M)$ contains the fully invariant subgroup of index $a^{2}$.

Proof. We can (cf. $\left.\left[\mathbf{H}_{2}\right]\right)$ represent $\pi_{1}(M)=\left\langle x, y: x^{p}=y^{q}\right\rangle$ where $\pi_{1}(\partial M)$ is freely generated by $\mu=x^{r} y^{-s}$ (where $p s-q r=1$ ), and $\lambda=x^{p}$ $=y^{q}$. Note that $\lambda$ is not a longitude in the traditional sense $(\lambda \notin$ $\left[\pi_{1}(M), \pi_{1}(M)\right]$ ), but, being central in $\pi_{1}(M)$, is more convenient to consider here. 
Let $Q$ be a finite group which contains noncommuting elements $\alpha, \beta$ which have orders $O(\alpha)=p, O(\beta)=q$, and with $O\left(\alpha \beta^{-1}\right)=a$. For example, the triangle group $T=T(p, q, a)$ contains a torsion free normal subgroup $N$ of finite index $\left[\mathbf{F}_{4}\right]$, and we could let $Q=T / N$. We define a homomorphism

$$
\eta: \pi_{1}(M) \rightarrow Q \times\left\langle\tau: \tau^{a}=1\right\rangle
$$

by putting $\eta(x)=\left(\alpha^{-q}, \tau^{-q}\right), \eta(y)=\left(\beta^{p}, \tau^{-p}\right)$ and noting that $\eta\left(x^{p}\right)=$ $\left(1, \tau^{-p q}\right)=\eta\left(y^{q}\right)$.

Now $\eta(\mu)=\left(\alpha^{-q r} \beta^{-p s}, \tau^{p s-r q}\right)=\left(\alpha \beta^{-1}, \tau\right)$ and $\eta(\lambda)=\left(1, \tau^{-p q}\right)$. Since $(p q, a)=1, O\left(\tau^{-p q}\right)=a$. Also $O\left(\alpha \beta^{-1}, \tau\right)=a$, and the proof is completed by reference to Lemma 2.3.

Similar arguments can be used to establish that certain other subgroups are in $\mathscr{P} \mathscr{F}_{M}(\partial M)$; however $\pi\left(\mu, \lambda^{c}\right) \notin \mathscr{P} \mathscr{F}_{M}(\partial M)$ for any $c$, since $\mu$ normally generates $\pi_{1}(M)$. We leave the exact structure of $\mathscr{P}_{\mathcal{F}}(\partial M)$ open.

Combining 2.4 and 2.1 we immediately obtain a proof of

2.5 COROLlary. Let $M_{1}$ and $M_{2}$ be torus knot spaces, $f: \partial M_{1} \rightarrow \partial M_{2} a$ homeomorphism, and put $M=M_{1} \cup_{f} M_{2}$. Then $\pi_{1}(M)$ is virtually representable to $\mathbf{Z}$.

Note that $\beta_{1}(M)>0$ if and only if $f$ takes the longitude of $M_{1}$ to the longitude of $M_{2}$.

For a general knot space $M$, it may not be possible to carry out such complete computations; however, if we fix a standard meridian, longitude pair $\{\mu, \lambda\}$ of generators for $\pi_{1}(\partial M)$ and if $H=\operatorname{gp}\left\{\mu^{a}, \mu^{b} \lambda^{c}\right\} \in$ $\mathcal{P} \mathscr{F}_{M}(\partial M)$, then there is a finite sheeted regular cover $p: \tilde{M} \rightarrow M$ with $p_{*} \pi_{1}(\tilde{M}) \cap \pi_{1}(\partial M)=H$. If $b=0$, the manifold $\tilde{M}$ must embed in a closed manifold $\hat{M}$, which is an (unbranched) cover of the branched a-fold cyclic cover $M_{a}$ of $S^{3}$ branched over the knot. Conversely, the finite covers of $M_{a}$ corresponding to normal subgroups of $\pi_{1}\left(M_{a}\right)$ which are invariant under the group $\mathbf{Z}_{a}$ of covering transformations give rise to groups $H \in \mathcal{P} \mathscr{F}_{M}(\partial M)$ of the form $H=\operatorname{gp}\left\{\mu^{a}, \lambda^{c}\right\}$.

If $H_{1}\left(M_{a}\right) \neq 0$, we can conclude that $\operatorname{gp}\left\{\mu^{a}, \lambda\right\} \in \mathscr{P} \mathscr{F}_{M}(\partial Z M)$; for take a finite sheeted abelian cover $\hat{M} \rightarrow M_{a}$ corresponding to a fully invariant subgroup of $\pi_{1}\left(M_{a}\right)$. Then $\lambda$ lifts to $\hat{M}$ (since $\lambda \in\left[\pi_{1}\left(M_{a}\right)\right.$, $\left.\left.\pi_{1}\left(M_{a}\right)\right]\right)$, but the covering of $M$ induced by $\hat{M}$ is nonabelian.

If $H_{1}(\hat{M}) \neq 0$ one can iterate this process by taking a finite sheeted abelian cover $\hat{M} \rightarrow \hat{M}$ corresponding to a fully invariant subgroup of 
$\pi_{1}(\hat{M})$. We can conclude that $\operatorname{gp}\left\{\mu^{a}, \lambda^{c}\right\} \in \mathcal{P} \mathscr{F}_{M}(\partial M)$ where $c$ is the order of $\lambda \bmod \pi_{1}(\hat{M})$.

The procedures developed in the next two sections are particularly suited to analyzing these poly-abelian covers. In $\$ 5$ we give some illustrative calculations in Example 5.2.

3. Presentations of $H_{1}(\tilde{M})$. We give an account of the procedure intoduced by Fox $\left[\mathbf{F}_{3}\right]$ for calculating the first homology group $H_{1}(\tilde{M})$ of a finite sheeted cover $\tilde{M} \rightarrow M$ of a finite $\mathrm{CW}$ complex $M$ in terms of a presentation of $\pi_{1}(M)$, and we give some techniques for simplifying its application to the case of abelian and dihedral covers which are well suited to the analysis of 3-manifolds as given in the next section.

We regard the symmetric group $S_{q}$ as either the group of permutations of $\{1,2, \ldots, q\}$ or, equivalently, as the subgroup of $\operatorname{Gl}(q, \mathbf{Z})$ of matrices which permute the standard basis elements. In either case the action is on the right and $\sigma \in S_{q}$ will denote either the function

$$
i \rightarrow(i) \sigma
$$

or the matrix

$$
\left(\delta_{(\iota) \sigma, J}\right)
$$

as the context dictates.

Given a covering space $p: \tilde{M} \rightarrow M$ with $q<\infty$ sheets there is a homomorphism

$$
\theta: \pi_{1}(M) \rightarrow S_{q}
$$

defined as follows. Put $H=p_{*} \pi_{1}\left(\tilde{M}, \tilde{m}_{1}\right)$ and label the right cosets of $H$ as $H=H_{1}, H_{2}, \ldots, H_{q}$. Then

$$
\text { (i) } \theta(g)=j \Leftrightarrow H_{l} g=H_{j} \text {. }
$$

It is a standard fact that $\theta$ is well defined up to conjugation, that image $\theta$ is a transitive subgroup of $S_{q}$, that $H=\theta^{-1}\left\{\sigma \in S_{q}:(1) \sigma=1\right\}$, and, in fact, that the correspondence

$$
(p: \tilde{M} \rightarrow M) \leftrightarrow \theta
$$

is a one-to-one correspondence between equivalence classes of $q$-sheeted coverings of $M$ with conjugacy classes of representations of $\pi_{1}(M)$ onto transitive subgroups of $S_{q}$. We call $\theta$ the permutational representation of $\pi_{1}(M)$ associated to $p: \tilde{M} \rightarrow M$, and call $\theta$ regular precisely when the cover is regular. 
A matrix $\left(\lambda_{i j}\right)(1 \leq i \leq n, 1 \leq j \leq m)$ with entries in a ring $\Lambda$ presents a (left) $\Lambda$-module $A$ if $A \cong \operatorname{coker}\left(\left(\lambda_{i j}\right): \Lambda^{m} \rightarrow \Lambda^{n}\right)$, i.e., if $A$ is isomorphic to the quotient of the free $\Lambda$-module on generators $\left\{u_{1}, \ldots, u_{n}\right\}$ by the submodule generated by $\left\{\Sigma_{j} \lambda_{i j} u_{j} ; 1 \leq i \leq m\right\}$.

Two matrices which are equivalent in the sense of $\left[\mathbf{F}_{2}\right]$ present isomorphic modules. For a presentation $\left\langle x_{1}, \ldots, x_{n}: r_{1}, \ldots, r_{m}\right\rangle$ of a group $G$, the equivalence class of the Jacobian matrix of free derivatives $J=$ $\left(\partial r_{l} / \partial x_{l}\right)$, with entries in $\mathbf{Z} G$, depends only on $G\left[\mathbf{F}_{2}\right]$. For $G=\pi_{1}(M)$ and $p: \tilde{M} \rightarrow M$ a regular covering space whose group of covering transformations is $T$, the image of $J$ over $\mathbf{Z} T$ is known to present $H_{1}\left(\tilde{M}, \phi^{-1}\left(m_{0}\right)\right)$ as a module over $\mathbf{Z T}[\mathbf{C}],\left[\mathbf{H}_{\mathbf{3}}\right],\left[\mathbf{T}_{\mathbf{r}}\right]$.

The following theorem is a variation of this result applicable to nonregular, finite coverings. The proof we give below is a modification of the one given for Theorem 2.5 of $\left[\mathbf{H}_{3}\right]$.

3.1 TheOREM. (Fox $\left[\mathbf{F}_{3}\right]$.) Let $p: \tilde{M} \rightarrow M$ be a $q$ sheeted $(q<\infty)$ covering of a finite $C W$ complex $M, \phi: \pi_{1}(M) \rightarrow S_{q}$ the associated permutational representation, and $\left\langle x_{1}, \ldots, x_{n}: r_{1}, \ldots, r_{m}\right\rangle$ any presentation of $\pi_{1}(M)$. Then the $n q \times m q$ matrix of integers $\theta\left(\partial r_{l} / \partial x_{J}\right)$ is a presentation matrix, over $\mathbf{Z}$, for

$$
H_{1}(\tilde{M}) \oplus \mathbf{Z}^{q-1}
$$

Alernate proof. The result is independent of the particular presentation of $\pi_{1}(M)$. We choose the presentation naturally associated with a cell structure for $M$ which, with no loss of generality, we may assume has a single 0 -cell $m_{0}$.

We then show that $\theta\left(\partial r_{l} / \partial x_{J}\right)$ is the matrix, in terms of certain bases, of the boundary map of the cellular chain complex

$$
\text { ə: } C_{2}(\tilde{M}) \rightarrow C_{1}(\tilde{M}) \text {. }
$$

It then follows immediately that $\theta\left(\partial r_{l} / \partial x_{j}\right)$ presents

$$
H_{1}\left(\tilde{M}, p^{-1}\left(m_{0}\right)\right) \cong H_{1}(\tilde{M}) \oplus \mathbf{Z}^{q-1} .
$$

To determine the appropriate bases we choose characteristic maps

$$
f_{j}^{1}:(I, \partial I) \rightarrow\left(M^{(1)}, m_{0}\right), \quad 1 \leq j \leq n,
$$

for the 1-cells of $M$ and

$$
f_{i}^{2}:\left(I^{2}, \partial I^{2}, 0\right) \rightarrow\left(M^{(2)}, M^{(1)}, m_{0}\right), \quad 1 \leq i \leq m,
$$

for the 2-cells of $M$. So $x_{J}=\left[f_{j}^{1}\right], r_{i}=\left[f_{l}^{2} \mid \partial I^{2}\right]$. 
We can label the points of $p^{-1}\left(m_{0}\right)$ as $\left\{\tilde{m}_{1}, \ldots, \tilde{m}_{q}\right\}$ where

$$
p_{*} \pi_{1}\left(\tilde{M}, \tilde{m}_{i}\right)=\left\{g \in \pi_{1}(M):(i) \theta(g)=i\right\} .
$$

We let $\tilde{e}_{j, l}^{1}\left(\tilde{e}_{i, k}^{2}\right)$ be the 1-cell (2-cell) of $\tilde{M}$ whose characteristic map is the lifting $\tilde{f}$ of $f_{j}^{1}\left(f_{i}^{2}\right)$ such that $\tilde{f}(0)=\tilde{m}_{l}\left(=\tilde{m}_{k}\right)$.

Regarding the $\left\{\tilde{e}_{j, l}^{1}\right\}$ and $\left\{\tilde{e}_{i, k}^{2}\right\}$ as bases for $C_{1}(\tilde{M})$ and $C_{2}(\tilde{M})$, respectively, and putting $\theta\left(\partial r_{i} / \partial x_{j}\right)=\left(a_{(i, k),(j, l)}\right)$, one must verify that

$$
\partial \tilde{e}_{(l, k)}^{2}=\sum_{(j, l)} a_{(l, k),(j, l)} \tilde{e}_{(j, l)}^{1}
$$

to complete the proof (see $\left[\mathbf{H}_{3}\right]$ for related details).

While Theorem 3.1 gives a complete description of $H_{1}(\tilde{M})$ as an abelian group its application is hindered by the fact that, even in relatively simple situations, the matrix $\theta\left(\partial r_{i} / \partial x_{j}\right)$ will be large and a straightforward analysis of the group - say by reduction to an equivalent diagonal matrix - will be tedious. We offer some simplifications of both theoretical and practical interest.

Recall that for a matrix $J$ with entries in a commutative ring $\Lambda$, the $i$ th elementary ideal $\mathcal{E}_{i}(J)$ is the ideal generated by all $(n-i) \times(n-i)$ minor determinants of $J$, where $n$ is the number of columns of $J$. If $\Lambda=\mathbf{Z}$ we identify an ideal with its nonnegative generator. The elementary ideal theorem asserts

3.2. If the matrix $J$ of integers presents the abelian group $A$ and $r=\min \left\{i: \mathcal{E}_{i}(J) \neq 0\right\}$ then

$$
A=\mathbf{Z}^{r} \oplus \mathbf{Z}_{\mathscr{E}_{r} / \mathscr{E}_{r+1}} \oplus \mathbf{Z}_{\mathscr{E}_{r+1} / \mathscr{E}_{r+2}} \oplus \cdots
$$

In particular, $r=\operatorname{rank} A$ and $\mathcal{E}_{r}$ is the order of the torsion subgroup $A_{T}$ of $A$.

Since similar matrices are equivalent, one can use linear algebra to help analyze the abelian group presented by a matrix. Usually this requires an extension of the coefficient ring. One should try to extend minimally to preserve as much information as possible. However, regarding extensions to $\mathbf{C}$ one can say:

3.3 LEMMA. Let the square matrix $J$ of integers present the abelian group $A=\operatorname{coker}\left(J: \mathbf{Z}^{n} \rightarrow \mathbf{Z}^{n}\right.$ ). Suppose $\operatorname{ker} J=\operatorname{ker} J^{n}$ (which holds if $J$ is similar, over $\mathbf{C}$, to a diagonal matrix) and let the characteristic polynomial of $J$ be $x^{k} g(x)$ where $g(0) \neq 0$. Then

(i) rank $A=k$ (the multiplicity of 0 as a characteristic root and)

(ii) $O\left(A_{T}\right)$ divides $g(0)$ (the product of the nonzero characteristic roots of $A$ ). 
Proof. The first conclusion is immediate from the fact that the rank of $A$ is the nullity of $J$.

For (ii) let

$$
V_{0}=\left\{v \in \mathbf{Z}^{n}: v J^{k}=0\right\} \quad \text { and } \quad V_{1}=\left\{v \in \mathbf{Z}^{n}: v g(J)=0\right\} .
$$

Then $V^{\prime}=V_{0} \oplus V_{1}$ is a subgroup of finite index in $V=\mathbf{Z}^{n}$ and $V_{0}=\operatorname{ker} J$.

If $V^{\prime}=V$ the conclusion holds with equality: $O\left(A_{T}\right)=g(0)=\operatorname{det} A$.

In general we have exact sequences

$$
\begin{gathered}
0 \rightarrow V J / V^{\prime} J \rightarrow V / V^{\prime} J \rightarrow A \rightarrow 0, \\
0 \rightarrow V^{\prime} / V^{\prime} J \rightarrow V / V^{\prime} J \rightarrow V / V^{\prime} \rightarrow 0 .
\end{gathered}
$$

From the first and the fact that $V J / V^{\prime} J$ is finite, we have

$$
O\left(A_{T}\right)=O\left(V / V^{\prime} J\right)_{T} / O\left(V J / V^{\prime} J\right)_{T},
$$

and from the second that

$$
O\left(V / V^{\prime}\right)_{T} \text { is a multiple of } O\left(V / V^{\prime} J\right)_{T} / O\left(V^{\prime} / V^{\prime} J\right)_{T} \text {. }
$$

But $V / V^{\prime} \cong V J / V^{\prime} J$, and since $J \mid V^{\prime}$ has the same characteristic polynomial as $J$ it follows as above that $O\left(V^{\prime} / V^{\prime} J\right)_{T}=g(0)$. Combining we get the desired result.

Note that the assumption on $J$ is necessary (e.g.

$$
J=\left(\begin{array}{ll}
0 & n \\
0 & 0
\end{array}\right)
$$

and equality in (ii) does not hold in general (e.g.

$$
\left.J=\left(\begin{array}{cc}
a & a c \\
a c & a c^{2}
\end{array}\right)\right) \text {. }
$$

Equality does hold in (ii) if $A$ is finite. The statement of Theorem 5.3.3 of [N] seems to be based on the assumption that equality holds in general, and is incorrect - see Example 5.6.

In applying Theorem 3.1 to abelian, regular coverings we let

$$
T=\left\langle t_{1}: t_{1}^{q_{1}}=1\right\rangle \times \cdots \times\left\langle t_{k}: t_{k}^{q_{k}}=1\right\rangle
$$

be an abelian group of order $q=q_{1} q_{2} \cdots q_{k}$, and note that under pointwise multiplication the character group

$$
T^{*}=\operatorname{Hom}\left(T, S^{1}\right)
$$

is a group isomorphic to $T$ under the correspondence which associates

$$
t=t_{1}^{a_{1}} \cdots t_{k}^{a_{k}} \in T
$$


with

$$
\psi \in T^{*},
$$

where $\psi\left(t_{j}\right)=\zeta^{q a_{j} / q_{j}}, \zeta=\exp (2 \pi i / q)$.

With this notation we have:

3.4 LEMMA. There is a regular, faithful representation

$$
\theta: T \rightarrow S_{q} \subset \mathrm{Gl}(q, \mathbf{Z})
$$

and for any $n$ a matrix $W \in \operatorname{Gl}(n q, \mathbf{Z}[1 / q, \zeta])$ such that if $J$ is any $n \times n$ matrix with entries in $\mathbf{Z T}$ then

$$
W \theta(J) W^{-1}=\left(\begin{array}{lll}
\ddots & & \\
& \psi(J) & \\
& & \ddots
\end{array}\right)
$$

is in block diagonal form where there are $q n \times n$ blocks with entries in $\mathbf{Z}[\zeta]$ corresponding to the q-choices of $\psi \in T^{*}$.

Proof. For $t \in T, \theta(t)$ will be the matrix of the map $\mathbf{Z} T \rightarrow \mathbf{Z T}$ defined by multiplication by $t$ in terms of the $\mathbf{Z}$-basis $T=\left\{t_{1}^{a_{1}} \cdots t_{k}^{a_{k}} ; 1 \leq a_{i} \leq q_{i}\right\}$ (given some ordering - which for computational purposes it is convenient to choose as lexicographical on the exponents on the generators).

Let $\Lambda=\mathbf{Z}[1 / q, \zeta] \subset \mathbf{C}$ and regard $\mathbf{Z} T \subset \Lambda T$. A $\Lambda$-basis for $\Lambda T$ is थ $=\left\{u_{\psi}: \psi \in T^{*}\right\}$ where

$$
u_{\psi}=\sum_{t \in T} \psi\left(t^{-1}\right) t
$$

and

$$
t=\frac{1}{q} \sum_{\psi \in T^{*}} \psi(t) u_{\psi}
$$

The action of $T$ on $\Lambda T$ in terms of this basis is given by

$$
t u_{\psi}=\psi(t) u_{\psi} \text {. }
$$

Now $J$ is the matrix, with respect to the standard basis, of a $\mathbf{Z T} T$-linear map $f:(\mathbf{Z T})^{n} \rightarrow(\mathbf{Z T})^{n}$ as well as of its natural extension $f^{*}:(\Lambda T)^{n} \rightarrow$ $(\Lambda T)^{n}$ as a $\Lambda T$-linear map.

If we use the basis $T$ on each $\Lambda T$ summand and order the resulting set lexicographically first by the ordering of the summands and secondly by our fixed ordering of $T$, we get an ordered $\Lambda$-basis for $(\Lambda T)^{n}$ with respect to which the matrix of $f^{*}$ is $\theta(J)$. 
If we use the basis $\mathcal{U}$ on each summand and order first by the order of the subscripts (as elements of $T^{*} \cong T$ ) and secondly by the ordering of the summands, we get an ordered $\Lambda$-basis for $(\Lambda T)^{n}$ with respect to which the matrix of $f^{*}$ is the block diagonal of the conclusion. The two matrices must be similar over $\Lambda$ and the proof is complete.

In applying Lemma 3.4 it is convenient to deal only with spaces whose fundamental group has a presentation whose deficiency (number of generators - number of relators) is nonnegative. In this case by adding trivial relators, and accordingly adding rows of zeros to the Jacobian, we may assume the Jacobian is square.

For cases of negative deficiency one could add columns of zeros, but this corresponds to adding free generators to the group and complicates the analysis somewhat, and we will not consider these cases. Note that the fundamental group of any compact 3-manifold has a presentation of nonnegative deficiency.

The matrices $\theta(J)$ and $W \theta(J) W^{-1}$ have the same rank and the same elementary ideals - as ideals of $\Lambda$. These ideals have integer generators which are uniquely determined modulo units of $\Lambda$. If integers $m_{1}, m_{2}$ generate the same ideal of $\Lambda$ then $m_{1} /\left(m_{1}, m_{2}\right)$ and $m_{2} /\left(m_{1}, m_{2}\right)$ divide some power of $q$. Thus we have, continuing to use the same notation,

3.5 THEOREM. If the finite abelian group $T$ is the group of covering transformations of a regular covering $p: \tilde{M} \rightarrow M$ and if the square matrix $J$ is the image over $\mathbf{Z T}$ of the Jacobian of some presentation of $\pi_{1}(M)$, then $\theta(J)$ presents $H_{1}(\tilde{M}) \oplus \mathbf{Z}^{q-1}$. The rank and the torsion numbers prime to $q=O(T)$ can be computed from the block diagonal $W \theta(J) W^{-1}$. In particular, if for $\psi \in T^{*}, \psi(J)$ has nullity $n(\psi)$ and $\mathcal{E}_{n(\psi)}(\psi(J))$ has generator $g(\psi)$ then

$$
\beta_{1}(\tilde{M})=1-q+\sum_{\psi} n(\psi) \quad \text { and } \quad O\left(H_{1}(\tilde{M})_{T}\right)=u \prod_{\psi} g(\psi),
$$

where $u$ is a unit of $\mathbf{Z}[1 / q, \zeta]$.

Proof. The action of $T$ on $\mathbf{Z T}$ is free and transitive. Thus the composition $\pi_{1}(M) \rightarrow T \stackrel{\theta}{\rightarrow} S_{q}$ must be the permutational representation associated to $p: \tilde{M} \rightarrow M$. By $3.1 \theta(J)$ presents $H_{1}(\tilde{M}) \oplus \mathbf{Z}^{q-1}$. But $\theta(J)$ and $W \theta(J) W^{-1}$ have the same nullity: $\Sigma n(\psi)$ and the same elementary ideals (as ideals in $\mathbf{Z}[1 / q, \zeta]$ ); the first nonvanishing one is generated by $\Pi g(\psi)$. The conclusion then follows from 3.2. 
Note that the elements $g(\psi)$ need not be (associate to) integers - only their product is. In cases where the $g(\psi)$, or certain subproducts, are integral, much stronger conclusions can be drawn (cf. Theorem 4.3).

3.6 Corollary. Let $\hat{T}=\left\{t \in T: t^{2}=1\right\}$ and let $\hat{M} \rightarrow M$ be the regular covering corresponding to $\operatorname{Ker}\left(\pi_{1}(M) \rightarrow T \rightarrow T / T^{2} \cong \hat{T}\right)$. Then

$$
\beta_{1}(\tilde{M}) \equiv \beta_{1}(\hat{M}) \bmod 2 ;
$$

in particular, if $o(T)$ is odd,

$$
\beta_{1}(\tilde{M}) \equiv \beta_{1}(M) \bmod 2 ;
$$

in fact if $o(T)$ is an odd prime then

$$
\beta_{1}(\tilde{M}) \equiv \beta_{1}(M) \quad \bmod o(T)-1 .
$$

Proof. The automorphism $\zeta \rightarrow \zeta^{-1}$ of $\Lambda$ takes $\psi(J)$ to $\psi^{-1}(J)$; thus $n(\psi)=n\left(\psi^{-1}\right)$ and $\Sigma\left\{n(\psi): \psi^{2} \neq 1\right\} \equiv 0 \bmod 2$. From 3.5 we have

$$
\beta_{1}(\tilde{M}) \equiv \sum\left\{n(\psi): \psi^{2}=1\right\}-o(T)+1 \bmod 2 .
$$

Applying 3.5 to $\hat{M}$ we see that

$$
\beta_{1}(\hat{M})=\sum\left\{n(\psi): \psi^{2}=1\right\}-o(\hat{T})+1 .
$$

Since $o(T) \equiv o(\hat{T}) \bmod 2$, the first part follows.

The second part is immediate, since if $T$ is odd, $\hat{M}=M$.

For the third one, note that if $q=o(T)$ is prime, $T^{*}=\left\{\psi_{l}: 0 \leq i \leq\right.$ $q-1\}$, where $\psi_{i}(t)=\zeta^{i}(t$ a generator of $T$ and $\zeta=\exp (2 \pi i / q))$. For $1 \leq i \leq q-1$ there is an automorphism of $\Lambda$ taking $\zeta$ to $\zeta^{i}$. Thus $n\left(\psi_{l}\right)=n\left(\psi_{1}\right)$ and

$$
\begin{aligned}
\beta_{1}(\tilde{M}) & =n\left(\psi_{0}\right)+n\left(\psi_{1}\right)(q-1)-q+1 \\
& \equiv \beta_{1}(M) \quad \bmod q-1 .
\end{aligned}
$$

Note that for $M$ a knot space ( $T$ cyclic), $\beta_{1}(M)=\beta_{1}(\hat{M})=1$; thus we can recover the result (Theorem 2.14 of $\left[\mathbf{G}_{0}\right]$ ) that the first betti number of any (unbranched) cyclic covering of a knot space is odd.

The proof of Lemma 3.4 can clearly be adapted to finding representations in other groups. We state below a version for the dihedral group:

$$
D_{2 q}=\left\langle s, t: s^{2}=t^{q}=1, s t s^{-1}=t^{-1}\right\rangle
$$

This is pertinent, since [Hi], [M] every closed, oriented 3-manifold is a 3-fold cover of $S^{3}$ branched over a knot corresponding to an irregular representation of the knot goup into $D_{6}$. 
We let $S$ and $T$ be the subgroups of $D_{2 q}$ generated by $s$ and $t$, respectively. Note that every element of $\mathbf{Z D}_{2 q}$ is uniquely expressible in the form

$$
f_{1}(t)+f_{2}(t) \cdot s
$$

where $f_{i}(t)$ is a polynomial of degree $\leq q$ in $t$.

As before we let $\zeta=\exp (2 \pi i / q)$.

3.7 LEMMA. There is a faithful representation

$$
\theta: D_{2 q} \rightarrow S_{q} \subset \mathrm{Gl}(q, \mathbf{Z})
$$

with $S=\theta^{-1}\{\sigma:(1) \sigma=1\}$, and for any $n$ there is a matrix $W \in$ $\mathrm{Gl}(n q, \mathbf{Z}[1 / q, \zeta])$ such that if $J=J(t)+J_{2}(t) \cdot s$ is any $n \times n$ matrix with entries in $\mathbf{Z} D_{2 q}$, then

$$
W \theta(J) W^{-1}
$$

$$
=\left[\begin{array}{cccccc}
J_{1}(1)+J_{2}(1) & 0 & 0 & \cdots & 0 & 0 \\
0 & J_{1}(\zeta) & 0 & \cdots & 0 & J_{2}(\zeta) \\
0 & 0 & J_{1}\left(\zeta^{2}\right) & \cdots & J_{2}\left(\zeta^{2}\right) & 0 \\
\vdots & & & & & \\
0 & J_{2}\left(\zeta^{q-1}\right) & 0 & \cdots & 0 & J_{1}\left(\zeta^{q-1}\right)
\end{array}\right]
$$

Proof. Let $\mathbf{Z}\left[D_{2 q} / S\right] \subset \Lambda\left[D_{2 q} / S\right]$ be the free $\mathbf{Z}$, respectively $\Lambda=$ $\mathbf{Z}[1 / q, \zeta]$, modules on the set $D_{2 q} / S$ of right cosets of $S$. For $g \in D_{2 q}$, $\theta(g)$ is the matrix of the action on $\Lambda\left[D_{2 q} / S\right]$ given by right multiplication by $g$ in terms of the basis

$$
\left\{s, s t, \ldots, s t^{q-1}\right\}
$$

So

$$
\theta(t)=\left[\begin{array}{ccccc}
0 & 1 & 0 & \cdots & 0 \\
0 & 0 & 1 & \cdots & 0 \\
\vdots & & & & \\
0 & 0 & 0 & & 1 \\
1 & 0 & 0 & \cdots & 0
\end{array}\right], \quad \theta(s)=\left[\begin{array}{ccccc}
1 & 0 & \cdots & 0 & 0 \\
0 & 0 & \cdots & 1 & 0 \\
\vdots & & & & \\
0 & 1 & \cdots & 0 & 0
\end{array}\right] .
$$

Let $\left\{u_{0}, \ldots, u_{q-1}\right\}$ be the basis for $\Lambda\left[D_{2 q} / S\right]$ defined by

$$
u_{a}=\sum_{b} \zeta^{-a b} s t^{b}
$$


Note that for $f_{1}(t)+f_{2}(t) s \in \mathbf{Z} D_{2 q}$,

$$
u_{a}\left(f_{1}(t)+f_{2}(t) s\right)=f_{1}\left(\zeta^{a}\right) u_{a}+f_{2}\left(\zeta^{a}\right) u_{q-a} .
$$

The proof is completed in the same way as that of Lemma 3.4.

3.8. CoROllary. Let $M$ be a knot space, $\left\langle x_{1}, \ldots, x_{n}: r_{1}, \ldots, r_{n-1}\right\rangle$ be a Writtinger presentation of $\pi_{1}(M)$, and $\eta: \pi_{1}(M) \rightarrow D_{6}$ an epimorphism with $\eta\left(x_{1}\right)=s$. Let $p: \tilde{M} \rightarrow M$ be the 3-fold irregular cover corresponding to $\eta^{-1}(S)$ and let $\hat{M}$ be the corresponding branched covering of $S^{3}$. Write the $(n-1) \times(n-1)$ matrix

$$
\eta\left(\begin{array}{ccc}
\frac{\partial r_{1}}{\partial x_{2}} & \cdots & \frac{\partial r_{1}}{\partial x_{n}} \\
\frac{\partial r_{n-1}}{\partial x_{2}} & \cdots & \frac{\partial r_{n-1}}{\partial x_{n}}
\end{array}\right)
$$

in the form $J=J_{1}(t)+J_{2}(t)$ s. Then the matrix $\theta(J)$ presents the abelian group $H_{1}(\hat{M}) \oplus \mathbf{Z}$, which is also presented, modulo 3-torsion, by the matrix

$$
\left[\begin{array}{ll}
J_{1}(\zeta) & J_{2}(\zeta) \\
J_{2}\left(\zeta^{2}\right) & J_{1}\left(\zeta^{2}\right)
\end{array}\right]
$$

$\zeta=\exp (2 \pi i / 3)$

Proof. In the matrix $\theta\left(\partial r_{l} / \partial x_{J}\right)$ which presents $H_{1}\left(\tilde{M}, p^{-1}\left(m_{0}\right)\right) \cong$ $H_{1}(\tilde{M}) \oplus \mathbf{Z}^{2}$, the first three columns correspond to lifts $\tilde{x}_{10}, \tilde{x}_{11}, \tilde{x}_{12}$ of the meridian $x_{1}$ - the first of which projects homeomorphically. Thus the branch relations giving $H_{1}(\hat{M})$ from $H_{1}(\tilde{M})$ can be expressed $\tilde{x}_{10}=0$, $\tilde{x}_{11}+\tilde{x}_{12}=0$. The cycle $x_{11}$ maps, under $\partial$ to a generator of a summand of

$$
\operatorname{ker}\left(H_{0}\left(p^{-1}\left(m_{0}\right)\right) \rightarrow H_{0}(\tilde{M})\right) \cong \mathbf{Z}^{2}
$$

Thus putting the relations $\tilde{x}_{10}=\tilde{x}_{11}=\tilde{x}_{12}=0$ or, equivalently, deleting the first three columns gives a presentation of $H_{1}(\hat{M}) \oplus \mathbf{Z}$ by $\theta(J)$.

By Lemma $3.7 \theta(J)$ is similar, over $\mathbf{Z}[1 / 3, \zeta]$, to

$$
\left[\begin{array}{ccc}
J_{1}(1)+J_{2}(1) & 0 & 0 \\
0 & J_{1}(\zeta) & J_{2}(\zeta) \\
0 & J_{2}\left(\zeta^{2}\right) & J_{1}\left(\zeta^{2}\right)
\end{array}\right]
$$

But $J_{1}(1)+J_{2}(1)$ with a column of zeros added presents $H_{1}(M) \cong \mathbf{Z}$; so $J_{1}(1)+J_{2}(1)$ is invertible over $\mathbf{Z}$ and the final part follows. 
4. Symmetric Heegaard splittings. In this section we show how certain symmetry assumptions on a 3-manifold $M$ combine with the results of $\S 3$ to yield additional information about the homology of the covers of $M$.

Recall that on the group ring $\mathbf{Z} G$ there is a conjunction defined by

$$
\overline{\sum n_{i} g_{i}}=\sum n_{i} g_{i}^{-1}
$$

$n_{i} \in \mathbf{Z}, g_{i} \in G$.

In $\left[\mathbf{H}_{3}\right]$ we showed that the fundamental group of each closed, oriented 3-manifold $M$ has a pair $P_{1}, P_{2}$ of presentation which are dual in the sense that their Jacobian matrices over $Z \pi_{1}(M)$ satisfy

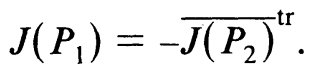

Since the Jacobians of any two presentations of $\pi_{1}(M)$ are equivalent, this duality yields useful information about fundamental groups of closed 3-manifolds (e.g. symmetry of elementary ideas).

If the dual presentations are related by some geometric symmetry, we can draw further information which is pertinent to the problems at hand.

First we note (relative to applying Theorem 3.1)

4.1 Lemma. Let $\theta: G \rightarrow S_{q} \subset \mathrm{Gl}(q, \mathbf{Z})$ be a homomorphism and $J$ an $n \times m$ matrix over $\mathbf{Z} G$. Then the $n q \times m q$ matrix $\theta(J)$, over $\mathbf{Z}$, satisfies

$$
\theta\left(\bar{J}^{\mathrm{tr}}\right)=\theta(J)^{\mathrm{tr}} \text {. }
$$

Proof. For $g \in G$ one computes that $\theta\left(g^{-1}\right)=\theta(g)^{\text {tr. }}$. Thus the conclusion describes the transposition of $\theta(J)$ obtained by first transposing in block form and then transposing each block.

The dual presentations mentioned above come from a Heegaard splitting (cf. $\left[\mathbf{H}_{1}\right]$, Chapter 2 ) of $M$ by alternately choosing (in a very particular way) the generators to come from one side of the splitting and the relators to come from the other. The symmetry we assume is that the sides can be interchanged. Specifically, we say that a Heegaard splitting $\left(V_{1}, V_{2}\right)$ of a 3-manifold $M$ is symmetric relative to the involution $\tau: M \rightarrow M$ if $\tau\left(V_{1}\right)=V_{2}$. The splitting will be called (+)-symmetric or (-)-symmetric according as deg $\tau=+1$ or $\operatorname{deg} \tau=-1$.

To construct examples, note that given a handle body $V_{1}$ and an involution $\tau_{o}: \partial V_{1} \rightarrow \partial V_{1}$, there is a uniquely determined Heegaard splitting $\left(V_{1}, V_{2}\right)$ which is symmetric relative to an extension $\tau: V_{1} \cup V_{2} \rightarrow$ $V_{1} \cup V_{2}$ of $\tau_{0}$. 
To detect symmetric Heegaard splittings in a more intrinsic way, it is helpful to think in terms of the quotient space $M / \tau$ and to consider cases according to the dimension of the fixed point set Fix $\tau$.

If Fix $\tau=\varnothing$, their $M / \tau$ is a 3-manifold and $p: M \rightarrow M / \tau$ is a covering map. The splitting of $M$ induces a one-sided Heegaard Splitting [Ru] of $M / \tau$, i.e., a union along the boundary of a handlebody and a twisted $I$-bundle over a closed surface (which will be oriented if and only if the splitting is (-)-symmetric). Conversely given a 3-manifold $M_{1}$ with a one-sided Heegaard splitting (which will exist, for example, if $M_{1}$ is orientable and $H_{1}\left(M ; \mathbf{Z}_{2}\right) \neq 0$ [Ru; Theorem 1]) there is a symmetric Heegaard splitting of the appropriate double cover of $M_{1}$. See Example 5.5 .

If $\operatorname{dim}(\operatorname{Fix} \tau)=1$, then $M / \tau$ is an orientable 3-manifold and $p$ : $M \rightarrow M / \tau$ is a branched covering. The manifold $M / \tau$ has the form $V /\{x=f(x)\}$, where $V$ is a handlebody, $\partial V=S_{0} \cup S_{1}$, where $S_{0}$ and $S_{1}$ are homeomorphic surfaces with $S_{0} \cap S_{1}=\partial S_{0}=\partial S_{1}$, and $f: S_{0} \rightarrow S_{1}$ is a homeomorphism (called the monodromy) such that $f \mid \partial S_{0}=1$.

If, in addition,

$$
\left(V ; S_{0}, S_{1}\right)=(S \times I ; S \times 0, S \times 1) /\{x \times I, x \in \partial S\}
$$

for some oriented surface $S$, then the structure on $M / \tau$ is called an open book decomposition, and $M$ has an open book decomposition whose monodromy is $f^{2}$. Conversely, if $M$ has an open book decomposition whose monodromy has a square root, then $M$ has a $(+)$-symmetric Heegaard splitting. The $2 k$-fold branched cyclic covers of $S^{3}$ branched over a fibered knot are such manifolds. See Example 5.2.

Note that every closed, oriented 3-manifold has an open book decomposition with connected binding [ $\mathbf{M} y]$.

If $\mathrm{dm}$ Fix $\tau=0$, then the splitting is (-)-symmetric and $M / \tau$ is not a manifold. The singular points have neighborhoods which are homeomorphic to the cone over $P^{2}$. The complement $N$ of these neighborhoods can be constructed as follows. Take an oriented surface $S$ with an even number, $2 k$, of boundary components. Take an $I$-bundle $W$ over $S$ which is twisted over each boundary component, a cube with $(1-k-\chi(S))$ handles, $V$, and identify $\partial V$ - $(k$ disks $)$ with the 0 -sphere bundle in $\partial W$ to obtain $N$. Then $M$ is the orientable double cover of $N$ with the $k 2$-sphere boundary components capped off with 3-cells. See Example 5.4.

If Fix $\tau \neq \varnothing$ we choose the basepoint $m_{0} \in$ Fix $\tau$; otherwise choose $m_{0} \in \partial V$ arbitrarily. For $\gamma: I \rightarrow \partial V_{1}$, a path from $m_{0}$ to $\tau\left(m_{0}\right)$, $\tau_{\gamma^{*}}: \pi_{1}\left(M, m_{0}\right) \rightarrow \pi_{1}\left(M, m_{0}\right)$ will denote the map

$$
\tau_{\gamma^{*}}([\omega])=\left[\gamma \cdot \tau \circ \omega \cdot \gamma^{-1}\right] \text {. }
$$


Note that $\left(\tau_{\gamma^{*}}\right)^{2}$ is conjugation by

$$
\alpha_{\gamma}=[\gamma \cdot \tau \circ \gamma]
$$

4.2 THEOREM. Let the closed, oriented 3-manifold $M$ have a Heegaard splitting $\left(V_{1}, V_{2}\right)$ which is symmetric relative to an involution $\tau: M \rightarrow M$. Then $\pi_{1}(M)$ has a presentation $P$ whose Jacobian matrix $J(P)$ over $\mathbf{Z} \pi_{1}(M)$ satisfies

$$
\tau_{\gamma^{*}} J(P)=(\operatorname{deg} \tau) \alpha_{\gamma} \overline{J(P)}^{\operatorname{tr}}
$$

for any path $\gamma$ from $m_{0}$ to $\tau\left(m_{0}\right)$.

Proof. This theorem extends Theorem 4.1 of $\left[\mathbf{H}_{3}\right]$ to which we refer for details.

Let $S=\partial V_{1}=\partial V_{2}$ and consider the diagram of inclusion induced maps

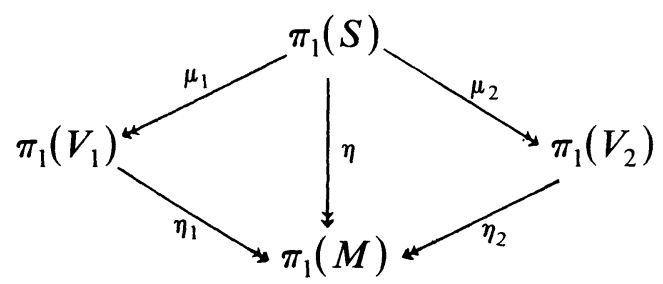

Put $N=\operatorname{ker} \eta$, let $p: \tilde{S} \rightarrow S$ be the regular cover corresponding to $N$, and identity $\pi_{1}(M)$ with the group of covering transformations.

We have the Reidemeister pairing

$$
\phi: N \times N \rightarrow \mathbf{Z} \pi_{1}(M)
$$

defined by

$$
\phi(u, v)=\sum_{\sigma \in \pi_{1}(M)}\langle h(u), \sigma h(v)\rangle \sigma,
$$

where $h: N \rightarrow H_{1}(\tilde{S})$ is defined by path lifting and the Hurewicz map and $\langle\rangle:, H_{1}(\tilde{S}) \times H_{1}(\tilde{S}) \rightarrow \mathbf{Z}$ is the integral intersection pairing. It is straightforward to compute (cf. Proposition 3.1 of $\left[\mathbf{H}_{3}\right]$ ) that $\phi$ is skew Hermitian bilinear, i.e.

$$
\begin{aligned}
\phi(u, v) & =-\overline{\phi(v, u)} \\
\phi\left(a_{1} u_{1} a_{1}^{-1} a_{2} u_{2} a_{2}^{-1}, v\right) & =a_{1} \phi\left(u_{1}, v\right)+a_{2} \phi\left(u_{2}, v\right) .
\end{aligned}
$$

Let $\left\{a_{1}, a_{2}, \ldots, a_{2 q}\right\}$ be a "standard basis" for

$$
\pi_{1}(S)=\left\langle a_{1}, \ldots, a_{2 g}: \prod\left[a_{2 i-1}, a_{2 i}\right]=1\right\rangle
$$


such that each $a_{2 l}(i=1, \ldots, g)$ contracts in $V_{1}$ and oriented so that $\left\langle a_{2 l}, a_{2 l-1}\right\rangle=+1$. Let $x_{l}=\mu_{1}\left(a_{2 i-1}\right)$, so $\left\{x_{1}, \ldots, x_{g}\right\}$ is a free basis for $\pi_{1}\left(V_{1}\right)$. Put $b_{j}=a_{2 \jmath-1} a_{2 j} a_{2 j-1}^{-1}$. Then as in the proof of 4.1 of $\left[\mathbf{H}_{3}\right]$, it follows from the formula of Theorem 3.3 of $\left[\mathbf{H}_{3}\right]$ that for any $v \in N$,

$$
\phi\left(v, b_{J}\right)=\eta_{1}\left(\frac{\partial \mu_{1}(v)}{\partial x_{J}}\right) .
$$

Let $\tau_{0}=\tau \mid S$ and put $r_{i}=\mu_{1} \tau_{0 \gamma^{*}}\left(b_{l}\right)$. Then $P=\left\langle x_{1}, \ldots, x_{n}: r_{1}, \ldots, r_{n}\right\rangle$ presents $\pi_{1}(M)$, and by the above formula,

$$
\tau_{\gamma^{*}} \eta_{1} \frac{\partial r_{l}}{\partial x_{J}}=\tau_{\gamma^{*}} \phi\left(\tau_{0 \gamma^{*}} b_{l}, b_{J}\right)
$$

Using the Hermitian properties of $\phi$ as well as naturality, the righthand side is in turn equal to

$$
\begin{aligned}
\left(\operatorname{deg} \tau_{0}\right) \phi( & \left.\tau_{0 \gamma^{*}}^{2} b_{l}, \tau_{0 \gamma^{*}} b_{j}\right) \\
& =\left(\operatorname{deg} \tau_{0}\right) \phi\left(\alpha_{\gamma} b_{i} \alpha_{\gamma}^{-1}, \tau_{0 \gamma^{*}} b_{j}\right) \\
& =-\left(\operatorname{deg} \tau_{0}\right) \alpha_{\gamma} \overline{\phi\left(\tau_{0 \gamma^{*}} b_{j}, b_{i}\right)}=-\left(\operatorname{deg} \tau_{0}\right) \alpha_{\gamma} \eta_{1}\left(\frac{\partial r_{l}}{\partial x_{l}}\right)
\end{aligned}
$$

and the proof is completed by the observation that $\operatorname{deg} \tau_{0}=-\operatorname{deg} \tau$.

We note that every term in the conclusion of Theorem 4.2 is, in general, necessary, as reference to Examples 5.4 and 5.5 will show. However, in many cases the effect of $\tau_{\gamma^{*}}$ and/or $\alpha_{\gamma}$ can be disregarded.

Suppose we have a homomorphism

$$
\nu: \pi_{1}(M) \rightarrow T
$$

to a finite abelian group $T$ such that $\operatorname{ker} \nu$ is invariant under $\tau_{*}$ and such that $\tau_{*}$ induces the identity of $T$. This would be the case, for example, if $\nu$ factored through $H_{1}(M / \tau)$. We will say that $\nu$ neutralizes the action of $\tau$.

Let $P$ be the presentation given by Theorem 4.2. Then

$$
\nu(J(P))=\operatorname{deg} \tau \nu(\alpha) \overline{\nu(J(P))}^{\mathrm{tr}} .
$$

Suppose further that $\nu(\alpha)$ has odd order, say $2 m+1$ (possibly $m=0)$. Then $\nu(J(P))$ is equivalent to $J_{1}=\nu\left(\alpha^{m} J(P)\right)$ and

$$
J_{1}=\operatorname{deg} \tau \bar{J}_{1}^{\mathrm{tr}} .
$$

Let $\theta: T \rightarrow S_{q}(q=o(T))$ be the representation of 3.4 ; so $\theta\left(J_{1}\right)$ presents $H_{1}(\tilde{M}) \oplus \mathbf{Z}^{q-1}$. But by $4.1 \theta\left(J_{1}\right)$ is either symmetric or skew symmetric according as $\operatorname{deg} \tau=+1$ or $\operatorname{deg} \tau=-1$. 
Moreover, in the block diagonal $W \theta\left(J_{1}\right) W^{-1}$ of 3.4 , the blocks will be complex (skew) Hermitian. From this we can conclude:

4.3 THEOREM. Let $M$ have a Heegaard splitting symmetric relative to an involution $\tau: M \rightarrow M$. If $p: \tilde{M} \rightarrow M$ is the regular covering corresponding to a $\tau$-neutralizing epimorphism $\nu: \pi_{1}(M) \rightarrow T$ to a finite abelian group $T$ such that $\nu(\alpha)$ has odd order, and $J_{1}$ is the (skew) Hermitian matrix described above, then the product of the nonzero eigenvalues of the block diagonal $W \theta\left(J_{1}\right) W^{-1}$ is an integer which is a multiple of $o\left(H_{1}(\tilde{M})_{T}\right)$ by a divisor of $o(T)^{m}$ for some $m$. Moreover:

(i) If $\operatorname{deg} \tau=-1$ then

$$
\beta_{1}(\tilde{M}) \equiv o(T)(n-1)+1 \bmod 2,
$$

when $n$ is the genus of the splitting, and the torsion subgroup of $H_{1}(\tilde{M})$ is a direct double: $H_{1}(\tilde{M})_{T} \cong A \oplus A$.

(ii) If $\operatorname{deg} \tau=+1$ and $o(T)$ is odd, then for any prime $p$ not dividing $o(T)$, the order of the p-primary component of $H_{1}(\tilde{M})$ is $p^{a+2 b}$ for some $b \geq 0$, where $p^{a}$ is the order of the p-primary component of $H_{1}(M)$.

Proof. $H_{1}(M) \oplus \mathbf{Z}^{q-1}(q=o(T))$ is presented by the $n q \times n q$ matrix $\theta\left(J_{1}\right)$ which is symmetric or skew symmetric according as deg $\tau=+1$ or $\operatorname{deg} \tau=-1$. In either case $\theta\left(J_{1}\right)$ is similar, over $\mathbf{C}$, to a diagonal matrix and the first part follows from Lemma 3.3. Note that this is a strengthening of 3.5 in that it gives a bound on the $q$-torsion part of $H_{1}(\tilde{M})_{T}$.

Part (i) follows from the fact (Theorems 8.6 and 32.2 of [Mc]) that a skew symmetric matrix of integers has even rank and is equivalent, over $\mathbf{Z}$, to one of the form

$$
\left[\begin{array}{cccccc}
0 & a_{1} & & & & \\
-a_{1} & 0 & & & & \\
& & 0 & a_{2} & & \\
& & -a_{2} & 0 & & \\
& & & & \ddots & \\
& & & & & 0
\end{array}\right]
$$

For part (ii) let, for each $\psi \in T^{*}, g(\psi)$ be the product of the nonzero eigenvalues of $\psi\left(J_{1}\right)$. Since $\psi(J)$ is complex Hermitian, $g(\psi)$ is real; so $g(\psi)=\overline{g(\psi)}=g\left(\psi^{-1}\right)$. For the trivial homomorphism $\psi_{0}$, we have $g\left(\psi_{0}\right)$ $=o\left(H_{1}(M)_{T}\right)$. Since $q$ is odd we can index $T^{*}-\left\{\psi_{0}\right\}=\left\{\psi_{1}, \ldots, \psi_{q-1}\right\}$ so $\psi_{i}^{-1}=\psi_{q-i}$. If $\boldsymbol{\sigma}$ is any automorphism of $\mathbf{Z}[1 / q, \zeta]$, then 
$\left\{\sigma \psi_{1}, \ldots, \sigma \psi_{(q-1) / 2}\right\}$ contains exactly one element from each pair $\left\{\psi_{i}, \psi_{q-i}\right\}$. Thus the product $P=g\left(\psi_{1}\right) \cdots g\left(\psi_{(q-1) / 2}\right)$ is invariant under each $\sigma$ and must, therefore, be an integer. The product of the nonzero eigenvalues of $W \theta\left(J_{1}\right) W^{-1}$ is $o\left(H_{1}(M)_{T}\right) \cdot P^{2}$, and (ii) follows.

Note that it may be the case that some $g(\psi)$ is divisible (in $\mathbf{Z}[1 / q, \zeta]$ ) by an integer, in which case further conclusions can be drawn. For example, if $q$ is an odd prime and the integer $R$ divides $g\left(\psi_{1}\right)$, then $R$ divides each $g\left(\psi_{i}\right)$. If $\left(R, o\left(H_{1}(M)_{T}\right)\right)=1$, then $R^{q-1}$ divides $o\left(H_{1}(\tilde{M})_{T}\right)$ (cf. Example 5.2, case $a=4$ ). I see no way to anticipate this in general.

Some cases of particular interest:

4.4. Corollary. If $M$ has a (-)-symmetric Heegaard splitting of odd genus relative to $\tau: M \rightarrow M$, then $\beta_{1}(M)$ is odd, as is $\beta_{1}(\tilde{M})$ for any regular cover $\tilde{M}$ corresponding to a finite, abelian representation of $\pi_{1}(M)$ which neutralizes the action of $\tau$.

4.5. COROLlaRY. If $M$ has a (-)-symmetric Heegaard splitting relative to $\tau: M \rightarrow M$ and $H_{1}\left(M / \tau ; \mathbf{Z}_{2}\right) \neq 0$, then $\pi_{1}(M)$ is virtually representable to $\mathbf{Z}$.

Proof. If $\tau$ is fixed point free then $M$ covers the nonorientable, closed 3-manifold $M / \tau$, so $\beta_{1}(M)>0$ (and the hypothesis $H_{1}\left(M / \tau ; \mathbf{Z}_{2}\right) \neq 0$ is redundant).

If Fix $\tau \neq \varnothing$ then we may have $\beta_{1}(M)=0$ (see Example 5.4). However, the map $\pi_{1}(M) \rightarrow \pi_{1}(M / \tau)$ is onto, since for $M_{0}=M-$ Fix $\tau$, $M_{0} \rightarrow M_{0} / \tau$ is a double covering and $\operatorname{ker}\left(\pi_{1}\left(M_{0} / \tau\right) \rightarrow \pi_{1}(M / \tau)\right)$ contains a nontrivial coset representative of $p_{*}\left(\pi_{1}\left(M_{0}\right)\right)$.

Thus the composition $\nu: \pi_{1}(M) \rightarrow H_{1}(M / \tau) \rightarrow \mathbf{Z}_{2}$ is onto and neutralizes $\tau$. Moreover, the element $\alpha$ of Theorem 4.2 is trivial (since Fix $\tau \neq \varnothing$ ), so 4.3(i) applies to the covering $p: \tilde{M} \rightarrow M$ corresponding to ker $\nu$ to show that $\beta_{1}(\tilde{M})$ is odd and, in particular, positive.

Note that, in contrast to 4.5 every lens space $L_{p, q}$ has a (+)-symmetric Heegaard splitting induced from a one-sided Heegaard splitting [Ru] of $L_{2 p, q}$ by the double covering $L_{p, q} \rightarrow L_{2 p, q}$ as in the discussion preceding Theorem 4.2.

5. Examples. Open book decompositions arise from branched cyclic coverings of fibered knots as described in $\$ 4$. They also provide good examples for analyzing the sets $\mathcal{P} \mathcal{F}_{M}(\partial M)$ as suggested at the end of $\S 2$. 
Accordingly, let $k$ be a knot in a closed 3-manifold $\Sigma$ (often, but not necessarily, $\Sigma=S^{3}$ ) such that the knot space $M=\overline{\Sigma-N(k)}$ fibers over $S^{1}$. Thus we can represent

$$
M=F \times I /(x, 0) \sim(f(x), 1),
$$

where $F$ is a surface with connected boundary and $f: F \rightarrow F$ is a homeomorphism which may be chosen so that $f \mid \partial F=1$, and so that for $x \in \partial F$ the curve $x \times I /(x, 0)=(x, 1)$ is a meridian (bounds a disk in $\Sigma-M)$.

For any positive integer $a$, the branched a-fold cyclic cover $p: M_{a} \rightarrow \Sigma$ of $\Sigma$ branched over $k$ has the open book decomposition with monodromy $f^{a}$.

5.1. LEMMA. $\pi_{1}\left(M_{a}\right)$ has the presentation

$$
P=\left\langle x_{1}, x_{2}, \ldots, f_{*}^{a}\left(x_{i}\right) x_{i}^{-1}=1, i=1,2, \cdots\right\rangle
$$

where $\left\{x_{1}, x_{2}, \cdots\right\}$ freely generates $\pi_{1}(F)$.

The Jacobian matrix of this presentation is

$$
f_{*}^{a-1}(L) \cdot f_{*}^{a-2}(L) \cdot f_{*}(L) \cdot L-I,
$$

where $L$ is the image, over $\mathbf{Z} \pi_{1}\left(M_{a}\right)$, of the "Jacobian matrix"

$$
\left(\frac{\partial f_{*}\left(x_{l}\right)}{\partial x_{J}}\right)
$$

of $f_{*}: \pi_{1}(F) \rightarrow \pi_{1}(F)$.

Proof. The first part is a direct application of Van Kampen's theorem. The second is a computation using the chain rule for free derivatives $\left[\mathbf{F}_{\mathbf{1}}\right.$, 2.6].

5.2. EXAMPLE. The figure eight knot space is fibered by the surface $F$ of genus one. We can represent $\pi_{1}(F)=\langle x, y$ : $\rangle$, where the monodromy $f$ induces

$$
\begin{aligned}
f_{*}: \quad x & \rightarrow x y^{-1}, \\
y & \rightarrow y^{2} x^{-1} .
\end{aligned}
$$

See Figure 1. So we have, using the notation of 5.1, that

$$
L=\left[\begin{array}{ll}
1 & -x y^{-1} \\
-y^{2} x^{-1} & 1+y
\end{array}\right] .
$$




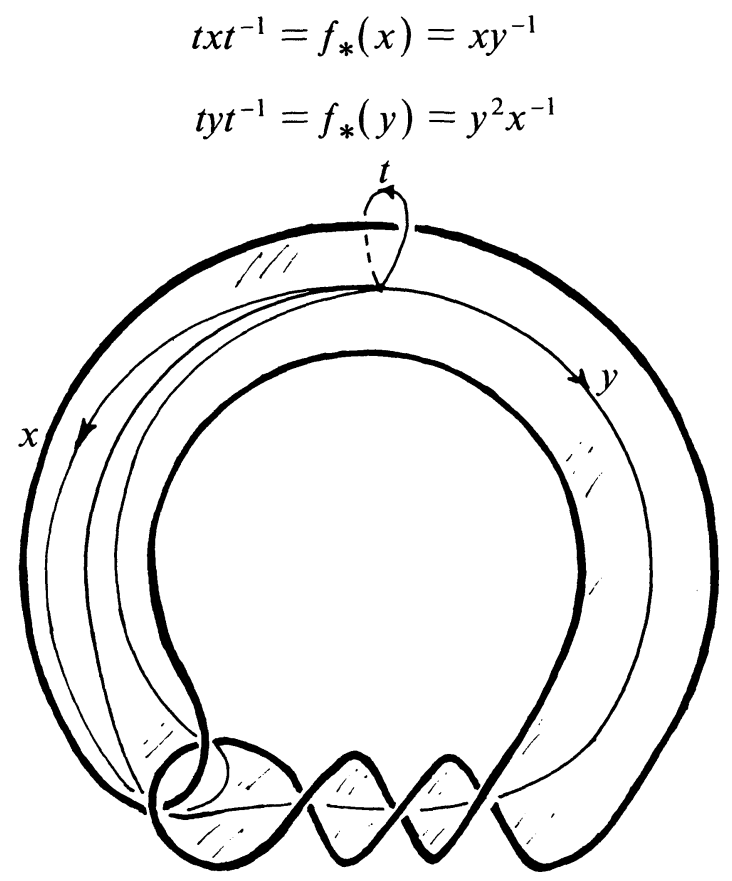

FIGURE 1. The figure eight knot

This must be taken in a different context for each value of $a$ since the entries are in $\mathbf{Z} \pi_{1}\left(M_{a}\right)$.

It is well known (and follows from 5.1) that $H_{1}\left(M_{a}\right)$ is presented by $L_{0}^{a}-I$, where

$$
L_{0}=\left[\begin{array}{cc}
1 & -1 \\
-1 & 2
\end{array}\right]
$$

is the image of $L$ under the trivial representation $x, y \rightarrow 1$

We investigate a few values of $a$.

$a=2$. $L_{0}^{2}-I=\left[\begin{array}{cc}1 & -3 \\ -3 & 4\end{array}\right]$ presents $\mathbf{Z}_{5}$. In fact $\pi_{1}\left(M_{2}\right) \cong \mathbf{Z}_{5}$, and it is not hard to show that $M_{2}$ is a lens space.

Thus we can conclude (as in the discussion at the end of \$2) that

$$
\operatorname{gp}\left\{\mu^{2}, \lambda^{c}\right\} \in \mathscr{P} \mathscr{F}_{M}(\partial M)
$$

if and only if $c=1$.

$$
a=3 . L_{0}^{3}-\mathrm{I}=\left[\begin{array}{cc}
4 & -8 \\
-8 & 12
\end{array}\right] \text { presents } \mathbf{Z}_{4} \oplus \mathbf{Z}_{4} \text {. For the 4-sheeted abelian }
$$
cover $\hat{M}_{3}$ of $M_{3}$ corresponding to

$$
\begin{aligned}
\operatorname{Ker}\left(\pi_{1}\left(M_{3}\right) \rightarrow H_{1}\left(M_{3}\right)\right. & \left.\rightarrow\left\langle s, t: s^{2}=t^{2}=1, s t=t s\right\rangle\right), \\
& x \rightarrow s, \\
y & \rightarrow t
\end{aligned}
$$


(which is fully invariant in $\pi_{1}\left(M_{3}\right)$ ), the Jacobian $f_{*}^{2}(L) \cdot f_{*}(L) \cdot L-I$ maps to

$$
J(s, t)=\left[\begin{array}{cc}
1+s+t+s t & -2(1+s+t+s t) \\
-2(1+s+t+s t) & 3(1+s+t+s t)
\end{array}\right] .
$$

Since $J(1,-1)=J(-1,1)=J(-1,-1)=\left[\begin{array}{ll}0 & 0 \\ 0 & 0\end{array}\right]$, we can conclude, from the latter part of Theorem 3.5 that $\beta_{1}\left(\hat{M}_{3}\right)=3$ and $o\left(H_{1}\left(\hat{M}_{3}\right)_{T}\right)$ is a power of 2.

Using the first part of Theorem 3.5, i.e. the $8 \times 8$ matrix $\theta(J)$, we see explicitly that $H_{1}\left(M_{3}\right)=\mathbf{Z}^{3}$.

From this we can see that $\pi_{1}\left(M_{3}\right)$ is virtually representable to $\mathbf{Z}$.

It is not hard to check that the longitude $\lambda$ lifts to a loop which represents a primitive element of $H_{1}\left(\hat{M}_{3}\right)$. The finite, abelian quotients of $\pi_{1}\left(\hat{M}_{3}\right)$ by fully invariant subgroups are of the form $\left(\mathbf{Z}_{c}\right)^{3}(c \in \mathbf{Z})$ in which $\lambda$ maps to an element of order $c$. By considering the corresponding covers of $\hat{M}_{3}$ we can deduce that

$$
\operatorname{gp}\left\{\mu^{3}, \lambda^{c}\right\} \in \mathscr{P} \mathcal{F}_{M}(\partial M)
$$

for all $c$.

$a=4 . \quad L_{0}^{4}-I=\left[\begin{array}{cc}12 & -21 \\ -21 & 33\end{array}\right]$ presents $\mathbf{Z}_{3} \oplus \mathbf{Z}_{3} \oplus \mathbf{Z}_{5}$. For the 5-sheeted abelian cover $\hat{M}_{4}$ of $M_{4}$ corresponding to the fully invariant subgroup

$$
\begin{aligned}
\operatorname{Ker}\left(\pi_{1}\left(M_{4}\right) \rightarrow\right. & \left.H_{1}\left(M_{4}\right) \rightarrow\left\langle t: t^{5}=1\right\rangle\right), \\
x & \rightarrow t, \\
y & \rightarrow t^{2},
\end{aligned}
$$

the Jacobian $f_{*}^{3}(L) \cdot f_{*}^{2}(L) \cdot f_{*}(L) \cdot L-I$ maps to

$$
J(t)=\left[\begin{array}{cc}
2 \phi(t)+2 t^{4} & -5 \phi(t)+2\left(1+t^{2}\right) \\
-5 \phi(t)+2\left(1+t^{4}\right) & 7 \phi(t)-2 t^{4}
\end{array}\right],
$$

where $\phi(t)=1+t+t^{2}+t^{3}+t^{4}$.

Now $M_{4}$ has a $(+)$-symmetric Heegaard splitting relative to the involution $\tau$ which is the square of the generator of the $\mathbf{Z}_{4}$ action on $M_{4}$. Our homomorphism $\pi_{1}\left(M_{4}\right) \rightarrow \mathbf{Z}_{5}$ neutralizes $\tau$ since it factors through $\pi_{1}\left(M_{2}\right)\left(M_{2}=M_{4} / \tau\right)$. Since Fix $\tau \neq \varnothing$, Theorem 4.3 applies. In particular, we have a Hermitian Jacobian which projects in $\mathbf{Z T}$ to

$$
J_{1}(t)=\left[\begin{array}{cc}
2\left(1+t+t^{4}\right) & t^{2}-\phi(t) \\
t^{3}-\phi(t) & -2\left(1+t^{2}+t^{3}\right)
\end{array}\right] .
$$

We give this for comparative purposes, without justification, except to note that it is not hard to show that $J(t)$ and $J_{1}(t)$ are equivalent over $\mathbf{Z T}$. 
Now for $\zeta=\exp (2 \pi i / 5)$,

$$
J_{1}(\zeta)=\left[\begin{array}{cc}
2\left(1+\zeta+\zeta^{4}\right) & \zeta^{2} \\
\zeta^{3} & -2\left(1+\zeta^{2}+\zeta^{3}\right)
\end{array}\right]
$$

has nullity 1 and its one nonzero eigenvalue (its trace) is

$$
2\left(\zeta^{4}+\zeta^{3}\right)-2\left(\zeta^{2}+\zeta^{3}\right)=2 \sqrt{5} .
$$

So, in the notation of $4.3, g\left(\psi_{1}\right)=g\left(\psi_{4}\right)=2 \sqrt{5}$. Similarly, $g\left(\zeta_{2}\right)=g\left(\zeta_{3}\right)$ $=-2 \sqrt{5}$ and $g\left(\psi_{0}\right)=3^{2} \times 5$. From the conclusion of 4.3 we can deduce that $\beta_{1}\left(\hat{M}_{4}\right)=0$ and $o\left(H_{1}(M)_{T}\right)=2^{4} \times 3^{2} \times 5^{k}(k=0,1)$.

If we used the matrix $J(t)$ and the statement of 3.5 , we can no longer use eigenvalues, since both eigenvalues of

$$
J(\zeta)=\left[\begin{array}{cc}
2 \zeta^{4} & 2\left(1+\zeta^{2}\right) \\
2\left(1+\zeta^{4}\right) & -2 \zeta^{4}
\end{array}\right]
$$

are zero. However, by row and column operations we can reduced $J(\zeta)$ to

$$
\left[\begin{array}{ll}
2 & 0 \\
0 & 0
\end{array}\right]
$$

Using the statement of the last part of 3.5 we can obtain only that the weaker conclusion that $o\left(H_{1}\left(\hat{M}_{4}\right)_{T}\right)=2^{4} \times 3^{2} \times 5^{k}$ for some $k \geq 0$. By using either of the $10 \times 10$ matrices $\theta(J)$ or $\theta\left(J_{1}\right)$, one can show explicitly that

$$
H_{1}\left(\hat{M}_{4}\right) \cong\left(\mathbf{Z}_{2}\right)^{4} \oplus\left(\mathbf{Z}_{3}\right)^{2}
$$

Finally, the longitude $\lambda$ lifts to a loop representing an element of order 2 in $H_{1}\left(\hat{M}_{4}\right)$, so we can conclude that

$$
\operatorname{gp}\left\{\mu^{4}, \lambda^{2}\right\} \in \mathcal{P} \mathscr{F}_{M}(\partial M) .
$$

$a=3 k$. It follows from Lemma 5.3 below that $M_{3 k}$ has a 4-sheeted regular cover $\hat{M}_{3 k}$ with $\beta_{1}\left(\hat{M}_{3 k}\right) \geq 3$. Actually $\beta_{1}\left(\hat{M}_{3 k}\right)=3$, since the $8 \times 8$ presentation matrix $\theta(J)$ for $H_{1}\left(\hat{M}_{3 k}\right) \oplus \mathbf{Z}^{3}$ can have nullity at most 6 because $H_{1}\left(M_{3 k}\right)$ is finite for all $k$. In fact, $H_{1}\left(\hat{M}_{3 k}\right)$ and $H_{1}\left(M_{3 k}\right)$ $\oplus \mathbf{Z}^{3}$ must be identical modulo 2-torsion. For example, $H_{1}\left(\hat{M}_{6}\right) \cong \mathbf{Z}^{3} \oplus$ $\mathbf{Z}_{285} \oplus$ 2-torsion.

Just as in the case $a=3$ we conclude that

$$
\operatorname{gp}\left\{\mu^{3 k}, \lambda^{c}\right\} \in \mathcal{P} \mathscr{F}_{M}(\partial M)
$$

for all $c$. 
5.3. LEMMA. Let $M_{a}$ be the branched a-fold cyclic cover of $S^{3}$ branched over a knot. Then for any $k \geq 1$ there is an epimorphism $\pi_{1}\left(M_{a k}\right) \rightarrow \pi_{1}\left(M_{a}\right)$. Thus for any finite sheeted cover $\hat{M}_{a} \rightarrow M_{a}$ there is a cover $\hat{M}_{a k} \rightarrow M_{a k}$ of the same index and an epimorphism $\pi_{1}\left(\hat{M}_{a k}\right) \rightarrow \pi_{1}\left(\hat{M}_{a}\right)$. So if $\pi_{1}\left(M_{a}\right)$ is virtually representable to $\mathbf{Z}$, then so is $\pi_{1}\left(M_{a k}\right)$.

Proof. For the associated unbranched covers $N_{a}, N_{a k}$ there is a $k$-fold cyclic covering $g: N_{a k} \rightarrow N_{a}$. The epimorphism $\pi_{1}\left(N_{a}\right) \rightarrow \pi_{1}\left(M_{a}\right)$ kills an element whose powers give a set of coset representations of $g_{*} \pi_{1}\left(N_{a k}\right)$ in $\pi_{1}\left(N_{a}\right)$. Thus $\pi_{1}\left(N_{a k}\right) \rightarrow \pi_{1}\left(M_{a}\right)$ is epic, and the first part follows. The rest is straightforward.

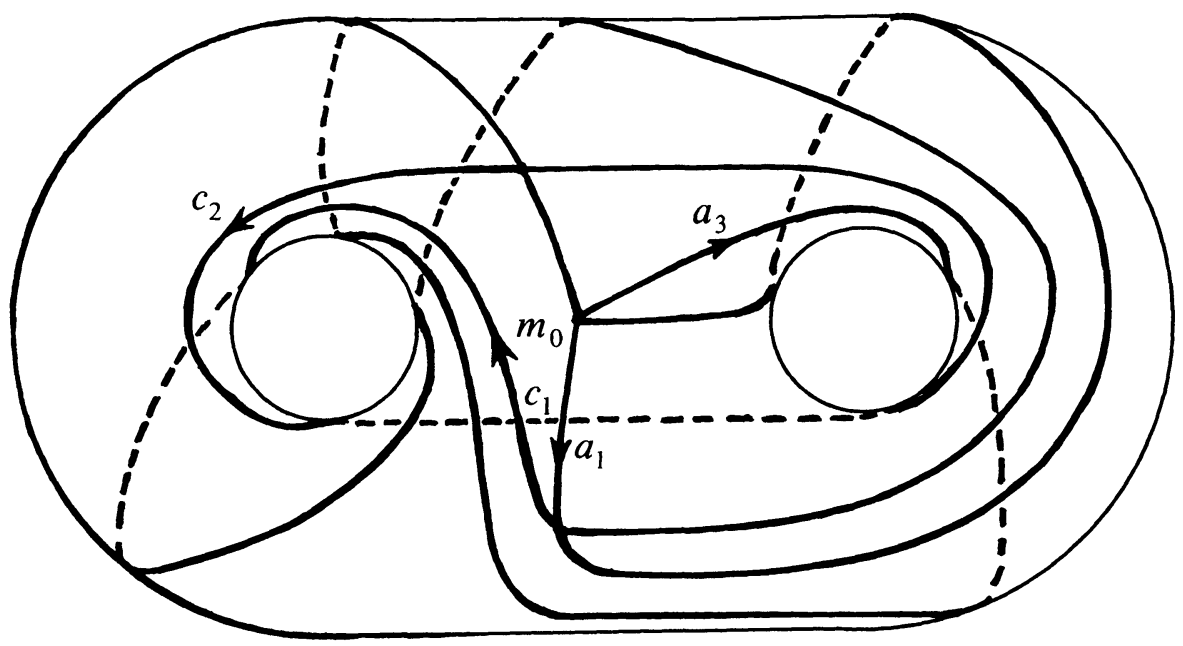

FIGURE 2

5.4. EXAmple. $A$ (-)-symmetric Heegaard splitting of genus 2 with Fix $\tau \neq \varnothing$ is described by Figure 2. This shows the splitting surface $S$, the curves $a_{1}, a_{3}$ (using the notation of the proof of Theorem 4.2) which represent generators $x_{1}, x_{2}$ for $\pi_{1}\left(V_{1}\right)$ and curves $c_{1}, c_{2}$ which are nullhomotopic in $V_{1}$. The curves $b_{1}, b_{2}$ are given by $b_{i}=d_{i} c_{i} d_{i}^{-1}$, where $d_{t}$ is the path which proceeds from the basepoint to the point $a_{2 i-1} \cap c_{i}$ along $a_{2 i-1}$ in the positive direction. Note that for any based loop in $S$ transverse to $c_{1}$ and $c_{2}$ the element in $\pi_{1}\left(V_{1}\right)$ represented by the loop can be read off from its intersection pattern with the $c_{i}$ 's - recording an $x_{i}$ $\left(x_{i}^{-1}\right)$ each time the loop crosses $c_{i}$ in the positive (negative) direction.

The involution $\tau_{0}: S \rightarrow S$ is a rotation of $180^{\circ}$ about an axis perpendicular to the paper and through the center of $S$. This extends to the involution $\tau: M \rightarrow M$ with $\tau\left(V_{1}\right)=V_{2}$ and $\operatorname{deg} \tau=-\operatorname{deg} \tau_{0}=-1$.

Since Fix $\tau \neq \varnothing$, we have no basepoint problem; so we take $\alpha=1$. 
The presentation of $\pi_{1}(M)$ is given with generators $x=x_{1}, y=x_{2}$ (to avoid subscripts) and relations $r_{1}=\mu_{1} \tau_{*}\left(b_{1}\right), r_{2}=\mu_{2} \tau_{*}\left(b_{2}\right)$. We can read these using the curves $\tau\left(d_{1} c_{1} d_{1}^{-1}\right), \tau\left(d_{2} c_{2} d_{2}^{-1}\right)$ as above to get:

$$
\begin{aligned}
& r_{1}=y x^{-1} y^{2} x y, \\
& r_{2}=\left(x^{2} y x y^{2}\right) x^{-1} y x^{-2} y^{-1} x^{-1}\left(x^{2} y x y^{2}\right)^{-1} .
\end{aligned}
$$

Caution: If one simplifies these relations (by cyclic reduction, say) then one is likely to loose the desired symmetry properties of the Jacobian matrix.

One can also read from the picture that

$$
\begin{aligned}
& \tau_{*}(x)=y^{-1} x^{-1} y^{-1} x^{-2}, \\
& \tau_{*}(y)=x^{2} y x y^{2} x^{-1} .
\end{aligned}
$$

The conclusion

$$
\tau_{*}\left(\frac{\partial r_{i}}{\partial x_{j}}\right)=-{\overline{\left(\frac{\partial r_{i}}{\partial x_{j}}\right.}}^{\mathrm{tr}}
$$

of 4.2 is valid, as can be seen by direct computation - although this becomes somewhat tedious, and one must remember that the relations may be needed, as this is a statement about $\mathbf{Z} \pi_{1}(M)$.

If we abelianize, we get

$$
H_{1}(M)=\left\langle x, y: x^{4}=y^{4}=1, x y=y x\right\rangle .
$$

The Jacobian reduces to

$$
J=\left[\begin{array}{cc}
x^{3}\left(y^{3}-y\right) & 1+x^{3} y+x^{3} y^{2}+y^{3} \\
-\left(1+x+x^{2} y^{3}+x^{3} y^{3}\right) & \left(x^{2}-1\right) y^{3}
\end{array}\right]
$$

and the effect of $\tau$ on $H_{1}(M)$ becomes

$$
\begin{gathered}
\tau_{*}(x)=x y^{2}, \\
\tau_{*}(y)=x^{2} y^{3} .
\end{gathered}
$$

It is elementary to check that

$$
\tau_{*} J=-\bar{J}^{\mathrm{tr}}
$$

The map $\nu: \pi_{1}(M) \rightarrow H_{1}(M) \rightarrow\left\langle x, y: x^{2}=Y^{2}=1, x y=y x\right\rangle$ neutralizes the action of $\tau$ and maps $J$ to the skew Hermitian matrix 


$$
\left[\begin{array}{cc}
0 & 1+x+y+x y \\
-(1+x+y+x y) & 0
\end{array}\right] .
$$

It is easy to check that the 4-sheeted covering $\tilde{M} \rightarrow M$ corresponding to ker $\nu$ has $H_{1}(\tilde{M}) \cong \mathbf{Z}^{3}$.

Thus $\pi_{1}(M)$ is virtually representable to $\mathbf{Z}$, as Theorem 4.5 guarantees even though $H_{1}(M)$ is finite.

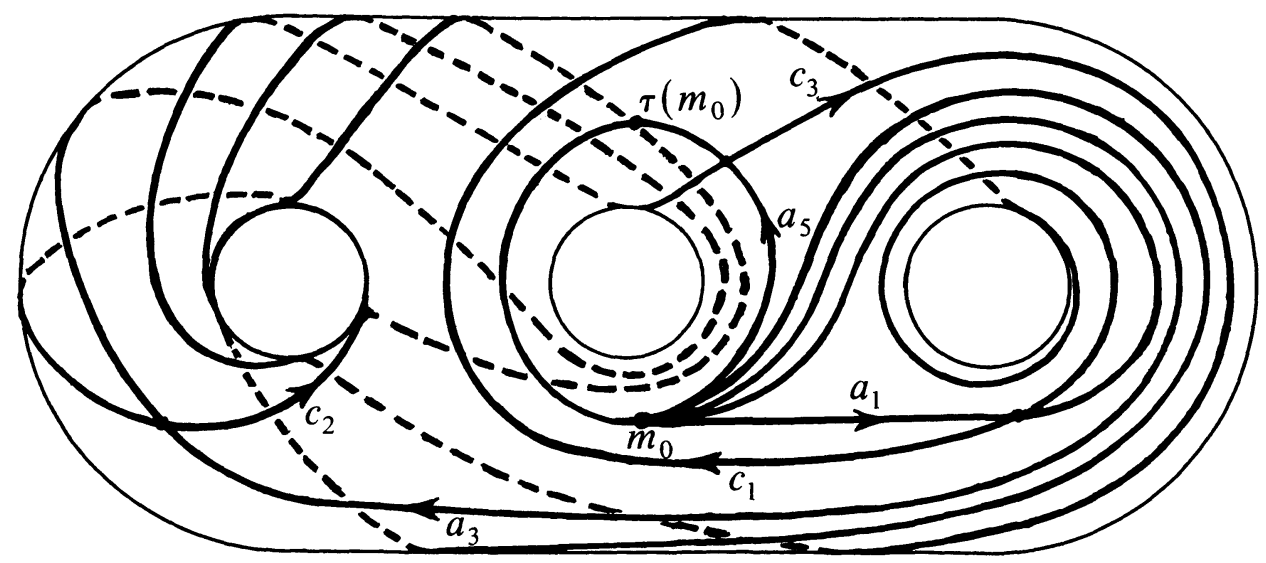

FIGURE 3

5.5. ExAmple. $A(-)$-symmetric Heegaard splitting of genus 3 with Fix $\tau=\varnothing$ is described by Figure 3. The procedure is the same as for Example 5.4, except in this example the involution $\tau_{0}: S \rightarrow S$ is a rotation of $180^{\circ}$ about an axis perpendicular to the paper and through the "center hole" of $S$.

The basepoint change is by the path $\gamma$ shown. The element $\alpha_{\gamma}=$ $[\gamma \cdot \tau(\gamma)]=x_{3}$.

The relations, in terms of generators $x=x_{1}, y=x_{2}, z=x_{3}$ given by $r_{i}=\mu_{1} \tau_{\gamma^{*}}\left(b_{l}\right)$ are

$$
\begin{aligned}
& r_{1}=x y^{2} z^{-1} y x^{-1} z^{-1} \\
& r_{2}=\left(z x y z^{-1} y\right) x^{-1} z^{-1} x^{-1} y^{-1} z y z^{-1} x^{-1}\left(z x y z^{-1} y\right)^{-1}, \\
& r_{3}=z x y z^{-1} y x z y^{-1} z^{-1} .
\end{aligned}
$$

Abelianization gives

$$
H_{1}(M)=\left\langle x, y, z: y=x^{-2}, z=x^{-3}\right\rangle=\langle x:\rangle .
$$

as 4.4 guarantees. The map $\tau_{\gamma^{*}}: x \rightarrow z x y^{-2} x^{-1} z^{-1}$, etc. reduces to the identity on $H_{1}(M)$, so every abelian representation neturalizes $\tau$. 
The abelianized Jacobian of this presentation is

$$
J=\left[\begin{array}{ccc}
1-x^{-3} & x^{-1}+1+x & -2 \\
-x^{-4}-x^{-3}-x^{-2} & x^{-3}-1 & -x^{-2}-x^{-1}+1 \\
2 x^{-3} & -x^{-3}+x^{-2}+x^{-1} & x^{-2}-x^{-1}
\end{array}\right]
$$

This is easily seen to satisfy

$$
J=-x^{-3} \bar{J}^{\mathrm{tr}}
$$

in accordance with 4.2 .

The $k$-sheeted cyclic cover $\tilde{M}_{k} \rightarrow M$ can be analyzed by 3.5 , the odd sheeted ones must satisfy 4.3(i). A few values are:

$$
\begin{aligned}
& H_{1}\left(\tilde{M}_{2}\right)=\mathbf{Z} \oplus \mathbf{Z}_{5}, \\
& H_{1}\left(\tilde{M}_{3}\right)=\mathbf{Z} \oplus\left(\mathbf{Z}_{4}\right)^{2}, \\
& H_{1}\left(\tilde{M}_{4}\right)=\mathbf{Z} \oplus \mathbf{Z}_{5} \oplus\left(\mathbf{Z}_{3}\right)^{2}, \\
& H_{1}\left(\tilde{M}_{5}\right)=\mathbf{Z} \oplus\left(Z_{11}\right)^{2} .
\end{aligned}
$$

5.6. EXAmple. The knot $8_{10}[\mathbf{R}]$ has Alexander polynomial $\Delta(t)=$ $\left(1-t+t^{2}\right)^{3}$, and it is straightforward to show that the abelianized Jacobian matrix of a presentation of the knot group is equivalent to $(0, \Delta(t))$.

The 6-fold cyclic cover $M_{6}$ of $S^{3}$ branched over $8_{10}$ has

$$
H_{1}\left(M_{6}\right) \cong \mathbf{Z}^{2} \oplus\left(\mathbf{Z}_{4}\right)^{2} \oplus \mathbf{Z}_{9}
$$

as can be computed from Theorem 3.4, together with the single branch relation which kills an $\infty$-cyclic summand.

However, for $\zeta=\exp (2 \pi i / 6), \Delta(\zeta)=\Delta\left(\zeta^{5}\right)=0, \Delta(1)=1$, and $\Delta\left(\zeta^{2}\right) \Delta\left(\zeta^{3}\right) \Delta\left(\zeta^{4}\right)=1728=12 o\left(H_{1}\left(M_{6}\right)_{T}\right)$. This shows that the statement of Theorem 5.3.3 of [N] is incorrect. Refer to Lemma 3.3 for the correct statement.

\section{REFERENCES}

[C] R. Crowell, Corresponding group and module sequences, Nagoya Math. J., 19 (1961), 27-40.

[CJR] M. Culler, W. Jaco and H. Rubinstein, Incompressible surfaces in once punctured torus bundles, Proc. London Math. Soc., (3), XLV (1982), 385-419.

[FH] W. Floyd and A. Hatcher, Incompressible surfaces in punctured torus bundles, Topology Appl., 13 (1982), 263-282. 
$\left[\mathrm{F}_{1}\right] \quad \mathrm{R}$. H. Fox, Free differential calculus, $I$, Derivation in the free group ring, Ann. of Math., (2) 57, 547-560.

$\left[\mathrm{F}_{2}\right] \quad$ Free differential calculus, II, the isomorphism problem of groups, Ann. of Math., (2) 59, 196-210.

$\left[\mathrm{F}_{3}\right] \quad$, Free differential calculus, III Subgroups, Ann. of Math., (2) 64, 407-419.

$\left[\mathrm{F}_{4}\right] \quad$, On Fenchel's conjecture about F-groups, Math. Tidsskr. B. (1952), 61-65.

[G] L. Goeritz, Die Betti'schen Zahlen der zyklischen Uberlagerungsraume der Knotenaussenraume, Amer. J. Math., 56 (1934), 194-198.

$\left[\mathrm{G}_{0}\right]$ C. M. Gordon, Knots whose branched cyclic coverings have periodic homology, Trans. Amer. Math. Soc., 168 (1972), 357-370.

[HT] A. Hatcher and W. Thurston, Incompressible surfaces in two bridge knot complements, preprint.

$\left[\mathrm{H}_{1}\right]$ John Hempel, 3-manifolds, Annals of Math. Studies No. 86 Princeton Univ. Press (1976).

$\left[\mathrm{H}_{2}\right] \quad$ A simply connected 3-manifold is $\mathrm{S}^{3}$ if it is the sum of a solid torus and the complement of a torus knot, Proc. Amer. Math. Soc., 15 (1964), 154-158.

$\left[\mathrm{H}_{3}\right] \quad$, Intersection calculus on surfaces with application to 3-manifolds, Memoirs Amer. Math. Soc., 43, No. 282 (1983).

[Hi] H. M. Hilden, Three-fold branched coverings of $S^{3}$, Amer. J. Math., 98 (1976), 989-997.

[HK] F. Hosokawa and S. Kinoshita, On the homology of branched cyclic coverings of knots and links, Osaka Math. J., 12 (1960), 331-355.

[J] W. Jaco, Lectures on 3-manifold Topology, C.B.M.S. Regional Conference Series No. 43, Amer. Math. Soc., (1980).

[JS] W. Jaco, and P. Shalen, Seifert fibered spaces in 3-manifolds, Memoirs of the Amer. Math. Soc., 21, No. 220, (1979).

[Jo] K. Johannson, Homotopy Equivalences of 3-manifolds with boundaries, Lecture Notes in Math. No. 761, Springer-Verlag (1979).

[Mc] C. C. MacDuffee, The Theory of Matrices, Chelsea Publishing Co., (1946).

[M] J. M. Montesinos, Three-manifolds as 3-fold branched covers of $S^{3}$, Quart J. Math. Oxford, 27 (1976), 85-94.

[My] Robert Myers, Open book decompositions of 3-manifolds, Proc. Amer. Math. Soc., 72 (1978), 397-402.

[N] L. P. Neuwirth, Knot Groups, Annals of Math. Studies No. 56, Princeton University Press (1965).

[R] D. Rolfson, Knots and Links, Mathematics Lecture Series 7, Publish or Perish Inc., Berkeley, Ca., 1976.

[Ru] H. Rubinstein, One sided Heegaard splittings of 3-manifolds, Pacific J. Math., 76 (1978), 185-200.

[S] J. Simon, Some classes of knots with Property P, Topology of Manifolds (Proc. Univ. of Georgia Inst. 1969), Markham, Chicago, 1970, 195-199.

[T] W. Thurston, The Geometry and Topology of 3-manifolds, Mimeographed notes, Princeton University.

[Tr] H. F. Trotter, Homology of group systems with applications to knot theory, Ann. of Math., 76 (1962), 464-498.

$\left[\mathrm{W}_{1}\right]$ F. Waldhausen, Gruppen mit Zentrum and 3-dimensionale Mannifaltigkeiten, Topology, 6 (1967), 505-517.

$\left[\mathrm{W}_{2}\right] \quad \ldots$, On irreducible 3-manifolds which are sufficiently large, Ann. of Math., 87 (1968), 56-88.

$\left[\mathrm{W}_{3}\right] \quad$ Recent results on sufficiently large 3-manifolds, Symposia in Pure Math., 32 (1978), 21-38. 
Received September 9, 1981. Research supported in part by NSF MCS 78-06116.

RICE UNIVERSITY

HousTON, TX 77001

AND

THE UNIVERSITY OF MICHIGAN

ANN ARBOR, MI 48109 



\section{PACIFIC JOURNAL OF MATHEMATICS \\ EDITORS}

DONALD BABBITT (Managing Editor)

University of California.

Los Angeles, CA 90024

Hugo Rossi

University of Utah

Salt Lake City, UT 84112

C. C. Moore and Arthur Ogus

University of California

Berkeley, CA 94720
J. DugundjI

Department of Mathematics

University of Southern California

Los Angeles, CA 90089-1113

R. FinN and H. Samelson

Stanford University

Stanford, CA 94305

\section{ASSOCIATE EDITORS}
R. ARENS
E. F. BECKENBACH
B. H. NeumanN
F. WOLF
K. YoshidA (1906-1982)

\section{SUPPORTING INSTITUTIONS}

UNIVERSITY OF ARIZONA

UNIVERSITY OF BRITISH COLUMBIA

CALIFORNIA INSTITUTE OF TECHNOLOGY

UNIVERSITY OF CALIFORNIA

MONTANA STATE UNIVERSITY

UNIVERSITY OF NEVADA. RENO

NEW MEXICO STATE UNIVERSITY

OREGON STATE UNIVERSITY
UNIVERSITY OF OREGON

UNIVERSITY OF SOUTHERN CALIFORNIA

STANFORD UNIVERSITY

UNIVERSITY OF HAWAII

UNIVERSITY OF TOKYO

UNIVERSITY OF UTAH

WASHINGTON STATE UNIVERSITY

UNIVERSITY OF WASHINGTON 


\section{Pacific Journal of Mathematics}

Vol. 112, No. $1 \quad$ January, 1984

Richard Blaine Barrar and Henry Loeb, Characterizing the divided difference weights for extended complete Tchebycheff systems $\ldots \ldots \ldots \ldots 1$

Harold Bennett and David John Lutzer, Generalized ordered spaces with

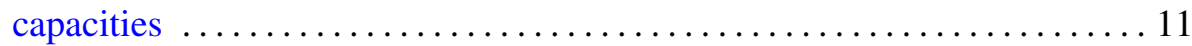

Geoffrey J. Butler and Lynn Harry Erbe, Comparison theorems for second-order operator-valued linear differential equations

Bohumil Cenkl and Richard D. Porter, de Rham theorem with cubical

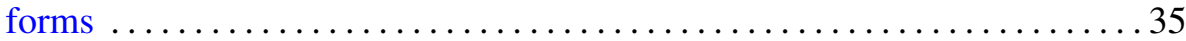

Zvonko Cerin, Characterizing global properties in inverse limits . ....... 49

Thomas Eugene Duchamp and Morris Kalka, Holomorphic foliations and deformations of the Hopf foliation .........................69 69

John Paul Hempel, Homology of coverings $\ldots \ldots \ldots \ldots \ldots \ldots \ldots \ldots \ldots$

Gerald Norman Hile and R. Z. Yeh, Inequalities for eigenvalues of the

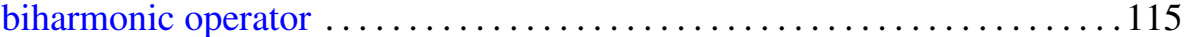

Kenneth Irwin Joy, A description of the topology on the dual space of a

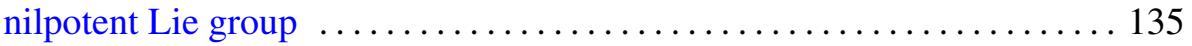

Alex Kumjian, On localizations and simple $C^{*}$-algebras $\ldots \ldots \ldots \ldots \ldots 141$

Bernardus de Pagter, The space of extended orthomorphisms in a Riesz

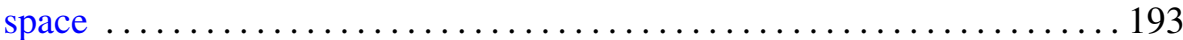

Stephen C. Persek, Iterated averaging for periodic systems with hidden

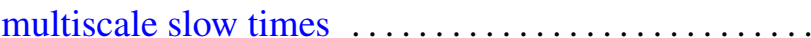

David Francis Rearick, Divisibility of arithmetic functions . . . . . . . . . 237

Masaaki Suzuki, The intrinsic metrics on the circular domains in $\mathbf{C}^{n}$ 